\title{
Upregulation of the Cdc42 GTPase limits replicative lifespan in budding yeast
}

Pil Jung Kang ${ }^{1}$, Rachel Mullner ${ }^{1}$, Haoyu Li $^{2}$, Derek Hansford ${ }^{3}$, Han-Wei Shen ${ }^{2}$, and Hay-Oak Park ${ }^{1 *}$

Department of Molecular Genetics ${ }^{1}$, Department of Computer Science and Engineering ${ }^{2}$, Department of Biomedical Engineering ${ }^{3}$, The Ohio State University, Columbus, OH 43210, USA

*Corresponding author: Department of Molecular Genetics, The Ohio State University, 484 West 12th Ave, Columbus, OH 43210-1292, E-mail: park.294@,osu.edu, Tel: 614-688-4575, Fax: 614-292-4466

Running Title: The Cdc42 GTPase and yeast aging

Key words: cell polarity, Saccharomyces cerevisiae, replicative lifespan, microfluidics, GTPaseactivating protein, cytokinesis remnants

Abbreviations: cytokinesis remnants (CRMs); replicative lifespan (RLS); GTPase-activating protein (GAP); wild type (WT); WGA (Wheat germ agglutinin)

Number of characters (Main text only): 15,041 (19,998 with Figure legends) 


\section{ABSTRACT}

Cell polarity underlies various cellular processes, including cell proliferation and asymmetric cell division. Cdc42, a conserved Rho GTPase, plays a central role in polarity establishment in yeast and animals. While cell polarity is critical for the asymmetric division of budding yeast, whether Cdc42 impacts lifespan is not clear. Here, we show by live-cell imaging that the active Cdc42 level is sporadically elevated in wild-type cells during repeated cell divisions but rarely in the long-lived bud8 deletion mutant. Remarkably, mild overexpression of $\mathrm{Cdc} 42$ causes premature aging with frequent symmetric cell divisions despite no harmful effects on young cells. Furthermore, deletion of $B U D 8$ rescues the short lifespan of an rgal mutant, which lacks a Cdc42 GTPase activating protein, and Bud8 competes with Rgal for localization to cytokinesis remnants. Collectively, our findings suggest that upregulation of $\mathrm{Cdc} 42$ is a proximal cause of cell death in old yeast cells and that Bud8 counteracts Rgal in modulating the Cdc42 activity to limit replicative lifespan.

\section{INTRODUCTION}

Individual cells of budding yeast undergo a finite number of cell divisions, while the yeast population is immortal in the presence of nutrients owing to asymmetric cell division. The irreversible arrest of cell proliferation (i.e., senescence) or death leads to the end of replicative aging. Replicative lifespan (RLS) is defined by the number of daughter cells a mother cell produces before death (Steinkraus et al., 2008). Each cell division leaves remnants - the 'birth scar' on the daughter cell wall and 'bud scar' on the mother cell wall (Barton, 1950; Mortimer and Johnston, 1959). Bud scars, also known as cytokinesis remnants (CRMs), are chitin-rich deposits that remained at the division sites. Since yeast cells bud at a new site that does not overlap with any previous bud site (Barton, 1950), the number of bud scars reflects the replicative age. Earlier reports postulated that depletion of available cell surface or nutrient exchange with the environment because of bud scars might limit RLS (Mortimer and Johnston, 1959; Johnston, 1966). However, multiple later studies disapproved of this idea because aging does not correlate with the amount of chitin on the cell wall. Furthermore, daughter cells from old mothers often have reduced lifespan despite the absence of bud scars in daughters (Egilmez and Jazwinski, 1989; Kennedy et al., 1994; Nestelbacher et al., 1999). Instead, aging appears to involve conserved regulatory systems across eukaryotic species (Kenyon, 2001; Wasko and Kaeberlein, 2014; McCormick et al., 2015).

Yeast cells undergo oriented cell division by selecting a specific bud site depending on their cell type. Haploid a or $\alpha$ cells bud in the axial pattern, in which both mother and daughter cells choose a new bud site adjacent to their immediately preceding division site. In contrast, $\mathbf{a} / \alpha$ cells (such as normal diploids) buds in the bipolar pattern, in which daughter cells bud preferentially at the pole distal to the division site, and mother cells can choose a bud site near either pole. The selection of a bud site depends on Bud proteins, which recruit and activate the Cdc42 GTPase module, determining the orientation of the polarity axis for budding (Bi and Park, 2012; Kang et al., 2014). Rga1, a Cdc42 GTPase-activating protein (GAP), is also involved in establishing the proper axis of cell polarity by preventing Cdc42 repolarization within the previous division site (Tong et al., 2007). Interestingly, cells lacking the negative polarity factors, including Rgal and Nba1, have a shorter RLS than wild type (WT) (Meitinger et al., 2014).

Some of the physiological changes observed during yeast aging, including budding pattern alteration, have been proposed to be a cause rather than a consequence of aging (Jazwinski et al., 
1998). However, there are somewhat conflicting views on the role of the budding pattern in aging. While Rsr1 (also known as Bud1, a Ras family GTPase involved in bud-site selection) has been suggested to be a longevity gene, theoretical modeling suggests that random budding is associated with longevity (Jazwinski et al., 1998; Jazwinski, 2001). Interestingly, a large-scale study of yeast deletion mutants has identified a bud8 8 mutant as one of the long-lived mutants (McCormick et al., 2015). Bud8 is involved in bipolar budding but has no known function in a or $\alpha$ cells (Zahner et al., 1996). Despite these intriguing pieces of information, whether Cdc42 or Bud proteins play any causative role in aging remains elusive. To address these outstanding questions, we performed genetic analysis and single-cell imaging for the entire lifespan of budding yeast. Our findings suggest that upregulation of $\mathrm{Cdc} 42$ is a proximal cause of senescence or death. We also propose that Bud8 and Rgal may oppositely impact lifespan by modulating the level or activity of $\mathrm{Cdc} 42$.

\section{RESULTS \& DISCUSSION}

60

Deletion mutants of the negative polarity factors, $\operatorname{rgal} \Delta$ and $n b a 1 \Delta$, often bud within the old cell division site, resulting in a narrow bud neck (Tong et al., 2007; Meitinger et al., 2014). This narrow bud neck phenotype has been implicated with the nuclear segregation defects and a cause of a shorter RLS of these mutants (Meitinger et al., 2014). Since Rax1 and Rax2, two interdependent integral membrane proteins that associate with CRMs (Chen et al., 2000; Kang et al., 2004), recruit Rgal via the Nba1-Nis1 complex (Miller et al., 2017), we asked whether rax1 $\Delta$ and rax $2 \Delta$ mutants also have a shorter RLS. RLS assays by micromanipulation indicated that $\operatorname{rax} 1 \Delta$ and $\operatorname{rax} 2 \Delta$ mutants have shorter RLS than WT (Fig. 1A) despite little growth defect during the exponential growth phase. However, we previously found that $\operatorname{rax} 1 \Delta$ and $\operatorname{rax} 2 \Delta$ cells are defective only in choosing the first bud site. Although daughter cells of $\operatorname{rax} 1 \Delta$ and $\operatorname{rax} 2 \Delta$ mutants often bud within the birth scar, subsequent buddings occur normally at a site adjacent to the previous division site (Miller et al., 2017). Unlike rgal $\Delta$, raxl $\Delta$ and $\operatorname{rax} 2 \Delta$ cells thus do not have the narrow bud neck. This observation raised the question of whether another defect of rgal $\Delta$ mutants may cause a shorter RLS.

We wondered whether the lack of (or reduced) Cdc42 GAP activity of rgal $1 \Delta$ mutants might result in a shorter RLS. Indeed, when we examined other Cdc42 GAP mutants rga $2 \Delta$ and bem $3 \Delta$, these mutants also displayed significantly reduced RLS than WT (Fig. 1B). The rga2 $\Delta$ and bem $3 \Delta$ cells do not bud within the previous division site and thus do not have a narrow bud neck (Tong et al., 2007). These observations suggest that Cdc42 activity, rather than the narrow bud neck, may limit RLS. We next considered another possibility that the bud-site selection defect of rgals mutants (Smith et al., 2002; Tong et al., 2007; Lo et al., 2013) might result in a shorter RLS. However, all three bud mutants we tested $-r \operatorname{sr} 1 \Delta$, axl1 , and $\operatorname{axl} 2 \Delta$ - had similar RLS as WT (Fig. 1C). On the other hand, a haploid bud8 $\Delta$ mutant has a longer RLS than WT (Fig. 1D), which confirms a previous finding (McCormick et $a l ., 2015)$. While $B U D 8$ is necessary for the bipolar budding pattern of $\mathbf{a} / \alpha$ cells, it is dispensable for the axial budding pattern of a or $\alpha$ cells (Zahner et al., 1996). The longer RLS of bud8 8 mutants is thus likely to be independent of its role in bud-site selection. Collectively, these observations suggest that any alteration of the budding pattern - i.e., the orientation of the polarity axis per se - is unlikely to affect replicative aging.

Since deletion of $B U D 8$ and $R G A 1$ has opposite effects on RLS, we next examined whether there is any functional interaction between these two genes in controlling RLS. We created a bud8 $\Delta$ rgal $\Delta$ double mutant and measured its lifespan together with both single mutants. Remarkably, the median 
RLS of the bud8 $\Delta$ rgal $\Delta$ mutant was similar to that of WT (Fig. 1D). A bud8 $\Delta$ rgal $\Delta$ mutant divided significantly more than that of an rgal $\Delta$ mutant before death $(+58.8 \%)$ but shorter than that of a bud $8 \Delta$ mutant (-20.6\%) (Fig. 1D). We next asked whether deletion of $B U D 8$ also rescues the same-site rebudding phenotype of rgal $\Delta$ mutants. We stained the bud8 $\operatorname{rgal} \Delta$ strain with WGA (Wheat germ agglutinin)-Texas Red to visualize the birth scar and bud scars. About $90 \%$ of bud8 $\Delta$ rgal $\Delta$ daughter cells had buds within the birth scar $(\mathrm{n}=80)$ (Fig. 1E), while a few mother cells had more than one bud scar within the birth scar. In contrast, no WT cells had bud scars or buds within the birth scar $(\mathrm{n}=80)$. Taken together, these results indicate that deletion of $B U D 8$ rescues a shorter RLS, but not the samesite re-budding phenotype, of an rgal $\Delta$ mutant. We thus conclude that the narrow bud neck resulting from the same-site re-budding phenotype is not the cause of a shorter RLS of rgal $\Delta$ mutants.

How does Bud8 interfere with Rgal's function and promote aging? Bud8 is known as a marker for the pole distal to the birth scar (i.e., distal pole) (Harkins et al., 2001) but is also observed at both poles of some unbudded cells (Taheri et al., 2000; Harkins et al., 2001). While Bud8 localization has been described in buds and newborn daughter cells, it has been overlooked in mother cells. To gain insight into Bud8's role in aging, we expressed Bud8 as an improved, functional mNeonGreen (mNG)tagged protein from its chromosomal locus. Interestingly, mNG-Bud8 localized to one pole or one side of the mother cell cortex of WT $\mathbf{a}$ and $\mathbf{a} / \alpha$ cells (in addition to the distal pole of growing buds, as previously reported) (Fig. 2A). Time-lapse imaging indicated that Bud8 at the bud tip is likely to remain at the distal pole in newborn cells after division, although the Bud8 signal was often weaker in mother cells that had undergone multiple cell divisions (Fig. S1).

Since Bud8 interacts with Rax1 (Kang et al., 2004), we speculated that Bud8 might localize to the distal pole and CRMs via its interaction with the Rax1-Rax2 complex. To test this idea, we examined the localization of Bud8 after staining cells with WGA-Texas Red. Bud8 localized to the distal pole and proximal pole (i.e., CRMs) in WT mother cells, although the signal was often weaker at the proximal pole (Fig. 2A). In the absence of Rax1, Bud8 localization was almost completely abolished at both poles of mother cells $(n=120)$, while it was mildly decreased in buds (Fig. 2, A \& C). Remarkably, Bud8 appeared in multiple puncta that overlapped with bud scars in WT mother cells that had divided several times (Figs. 2B \& S1, b). This surprising localization pattern of Bud8 to multiple CRMs was reminiscent of those of Rax1 and Rax2 (Chen et al., 2000; Kang et al., 2004). Indeed, time-lapse imaging showed that Bud8 exhibits significant colocalization with Rax2 in mother cells (Pearson's correlation coefficient $r=0.72$ ) (Figs. 2E \& S1, b). These results suggest that Bud8 is recruited to mother cells via Rax1-Rax2 at the distal pole and CRMs.

As discussed above, suppression of a short RLS of rgal $\Delta$ mutants by bud $8 \Delta$ suggests antagonistic roles of Bud8 and Rgal in replicative aging (see Fig. 1). The new localization pattern of Bud8 in mother cells raises the possibility that Bud8 and Rgal may compete for their localization to CRMs in mother cells. To test this possibility, we compared the localization of Bud8 in WT and rgal $\Delta$ cells (Fig. 2A). While the number of cells with localized Bud8 at each pole of rgal $\Delta$ mother cells was slightly higher ( $\mathrm{n}>120$, each strain; Fig. $2 \mathrm{C}$ ), the mNG-Bud8 level at the distal pole was significantly higher in rgal $\Delta$ mother cells than in WT mother cells $(p=0.003)$ (Fig. 2D). These results suggest that Bud8 competes with Rga1 for association with Rax1-Rax2 in mother cells.

Since the GAP Rgal inhibits Cdc42 at CRMs (Miller et al., 2017), the competition between Bud8 and Rga1 could result in an elevation of Cdc42-GTP at the mother cell cortex by hindering Rga1 
139 function. We tested this idea by imaging WT and bud8 $\Delta$ cells in a microfluidic platform. Microfluidics imaging allows for monitoring subcellular changes of single cells until death by washing away newborn daughter cells with continuous media flow (Xie et al., 2012; Chen et al., 2017). Calcofluorstaining of bud scars indicated that the age of the WT and bud8 cells initially trapped in the microfluidics imaging chambers was about the same (average age $=1.2 ; n>100$ ). We used a custommade program (see Figs. S2; Materials and Methods) or manually inspected the microfluidic images to count the number of cell divisions of each cell until death or senescence. On average, the bud8 $\Delta$ cells have undergone more cell divisions than WT before death or senescence, consistent with its longer RLS estimated by micromanipulation assays (see Fig. 1D).

We imaged the WT and bud8 8 strains expressing PBD-RFP, which interacts specifically with Cdc42-GTP (Okada et al., 2013; Okada et al., 2017), and Cdc3-GFP (a septin subunit) as a marker for the cell division site (see Fig. S2 \& Video S1). We then compared the PBD-RFP level during the G1 phase at each age of individual cells, as shown in single-cell trajectories (Fig. 3, A \& B). Each cell division is grouped based on the PBD level by highlighting the pixels above the basal level in young cells (see Fig. 3C; Materials and Methods). This analysis revealed sporadic elevation of Cdc42-GTP at the WT mother cell cortex during repeated cell divisions $(70 \%, \mathrm{n}=40$; Fig. 3A; Video S1). In contrast, such an elevation of Cdc42-GTP was rare in bud8 $\Delta$ mother cells: less than $25 \%$ of bud8 8 cells exhibited a modest Cdc42-GTP accumulation briefly during their entire lifespan ( $\mathrm{n}=40$; Fig. 3B). This difference in the PBD elevation between WT and bud8 $\Delta$ cells was also evident when we compared the number of cell divisions with high PBD levels (Fig. 3D). These observations support the idea that Bud 8 contributes to replicative aging by promoting the elevation of Cdc42-GTP at the mother cell cortex.

A shorter RLS of Cdc42 GAP mutants and lower Cdc42-GTP level in the long-lived bud8 $\Delta$ mutants raise the possibility that upregulation of $\mathrm{Cdc} 42$ during aging may cause a failure in polarity establishment and lead to irreversible growth arrest. Consistent with this possibility, mathematical modeling on spontaneous cell polarization predicted that a fraction of polarized cells decreases when the number of Cdc42 molecules in a cell increases (Altschuler et al., 2008). To test this possibility, we examined the impact of Cdc42 overexpression on RLS. We performed microfluidic imaging of a strain that carries extra copies of GFP-CDC42 (CDC42ov) on the chromosome. This CDC42ov strain grew equally well as WT (with the endogenous, untagged $C D C 42$ gene) at a temperature up to $34^{\circ} \mathrm{C}$ and grew better than the strain carrying a single-copy, partially functional GFP-CDC42 (Wu et al., 2015). The GFP-Cdc42 level was moderately higher (on average 2.2-fold) in this CDC42ov strain than the GFP-CDC42 strain (Fig. 4, Aa). We compared RLS of CDC42ov to WT as a control instead of the $G F P-C D C 42$ strain, which was a little sick at $30^{\circ} \mathrm{C}$. Both WT and $C D C 42 o v$ strains also expressed Cdc3-mCherry to facilitate automatic counting of cell divisions by a Python program (see Fig. S2; Materials and Methods). Strikingly, the $C D C 42 \mathrm{ov}$ strain underwent a significantly fewer number of cell divisions than WT before death $(\mathrm{n}=70$, WT; $\mathrm{n}=90$, CDC42ov; Fig. 4, Ab). These analyses suggest that even moderate elevation of Cdc42 is detrimental to older cells despite its little harmful effect on young cells.

Microfluidic imaging also uncovered distinct characteristics of aging cells. The GFP-CDC42ov cells often exhibited enlarged, round cell shapes without $\mathrm{Cdc} 42$ enrichment at a single site near senescence or death, indicating loss of cell polarity. Mothers sometimes produced daughter cells that were significantly larger in the last $20 \sim 30 \%$ of their lifespan, resulting in daughters about the same size as (and occasionally even bigger than) the mother at the time of division $(20 \% ; n=40)$. Symmetric cell 
186 division was also evident in their lifespan as these newborn daughter cells died at their first few cell divisions or even earlier than their mothers (Fig. 4, Ba). The GFP-Cdc42 level on the plasma membrane and endomembrane in these cells was significantly elevated during the last $30 \%$ of their lifespan (Fig. 4B, a \& c). Symmetric cell division and the elevation of Cdc42 were more common in cells that divided fewer than the average RLS of the GFP-CDC42ov strain. In contrast, in the relatively longer-lived cells in the same population $(15 \% ; n=40)$, Cdc42 elevation at old ages was minor (about 2 -fold), and the characteristics of symmetric cell division were rarely observed (Fig. 4B, b \& c). Some of these characteristics of the $C D C 42 \mathrm{ov}$ cells are remarkably similar to the observations on old yeast mother cells described in an earlier report (Kennedy et al., 1994), suggesting that Cdc42 overexpression causes premature aging.

Our data presented in this study suggest that Cdc42 elevation is a proximal cause of replicative aging in budding yeast. While $\mathrm{Cdc} 42$ is essential for the proliferation of young cells, its elevation and hyperactivation may cause senescence in older cells. This finding is consistent with the 'antagonistic pleiotropy' hypothesis (Williams, 1957). This evolutionary theory of senescence posits that alleles may have opposite pleiotropic effects at different ages. If the beneficial effects of such alleles early in life outweigh their deleterious effects at an old age, then such genetic variants would be favored and enriched in a population (Williams, 1957; Gaillard and Lemaître, 2017). It is noteworthy that Cdc42 has also been implicated in the senescence of mammalian cells. Notably, Cdc42 activity is substantially increased in hematopoietic stem cells and tissues from aged mice compared to those taken from young mice. This increased $\mathrm{Cdc} 42$ activity correlates with the depolarized phenotype, symmetric cell divisions, and aging in stem cells (Giebel, 2008; Florian et al., 2012; Florian et al., 2018). Consistent with a shorter RLS with the yeast Cdc42 GAP mutants (see Fig. 1, B \& D), loss of Cdc42 GAP has also resulted in premature senescence in mammalian cells (Wang et al., 2007). Thus, upregulation of $\mathrm{Cdc} 42$ may be a general cause of senescence in asymmetrically dividing cells. We propose that the balance between Bud8 and negative polarity factors such as Rga1 may determine RLS in budding yeast by controlling the Cdc42 activity in mother cells (Fig. 5). The competition between Bud8 and Rga 1 may account for the sporadic elevation of Cdc42-GTP during repeated cell divisions, and young cells seem to have an ability to adapt to such changes (see Fig. 3). While further investigation is required to understand the mechanisms, it is tempting to speculate that the stochastic elevation or activation of $\mathrm{Cdc} 42$ due to the competition of positive and negative polarity signaling may be one of the factors leading to the heterogeneity of lifespan among genetically identical cells in a population.

\section{MATERIALS AND METHODS}

\section{Yeast strains and growth conditions}

Standard methods of yeast genetics, DNA manipulation, and growth conditions were used (Guthrie and Fink, 1991). Yeast strains were grown in the appropriate synthetic medium containing $2 \%$ dextrose as a carbon source. Yeast strains used in this study are listed in Supplemental Table S1 with a brief description of construction methods. All yeast strains used for imaging express tagged proteins under their native promoters on the chromosomes. For imaging, yeast strains were grown at $30^{\circ} \mathrm{C}$, unless indicated otherwise, using the appropriate synthetic complete medium containing $2 \%$ dextrose as a carbon source.

\section{Determination of RLS}

Life span analyses were carried out by using micromanipulation as described previously (Steffen et al., $2009)$ using YPD plates containing (2\% [wt/vol] glucose and $2 \%$ [wt/vol] agar). During the 
233 intervening time of each micromanipulation, plates were sealed with parafilm and incubated at $30^{\circ} \mathrm{C}$.

234 Plates were shifted to $4^{\circ} \mathrm{C}$ for overnight incubation. Statistical significance of RLS data sets was analyzed by the Gehan-Breslow-Wilcoxon test and Log-rank (Mantel-Cox) test. For all strains tested, mean RLS and $p$ values were calculated from pooled experiments in which each strain of interest was compared with its respective WT strain. RLS was also estimated by counting the number of cell divisions from microfluidic images. A custom-made Python program was used for the analysis of properly oriented cells during imaging for the entire lifespan (see below). The number of cell divisions was manually counted with a visual inspection of microfluidic images of cells, whose positions were shifted relative to the pillars during imaging.

\section{Microscopy and image analysis}

Cells were grown in an appropriate synthetic medium overnight and then freshly subcultured for 3-4 h in the same medium. For most time-lapse imaging, images were captured ( $9 \mathrm{z}$ stacks, $0.4 \mu \mathrm{m}$ step) every 10 or 15 min with cells either mounted on a $\%$ agarose slab or a glass-bottomed dish (MatTek) containing the indicated medium with $5 \mu \mathrm{M}$ propyl gallate (Sigma), an anti-fade reagent, as previously described (Kang et al., 2018; Miller et al., 2019). The slab or dish was put directly on a stage (at 25$26^{\circ} \mathrm{C}$ ) or in a temperature-control chamber set to $30^{\circ} \mathrm{C}$, as indicated. Imaging involving mNG-Bud8 (Figs. 2 \& S1) was performed using a spinning disk confocal microscope (Ultra-VIEW VoX CSU-X1 system; Perkin Elmer-Cetus) equipped with a 100x /1.45 NA Plan-Apochromat Lambda oil immersion objective lens (Nikon); 440-, 488-, 515- and 561-nm solid-state lasers (Modular Laser System 2.0; Perkin Elmer-Cetus); and a back-thinned EM CCD (ImagEM C9100-23B; Hamamatsu Photonics) on an inverted microscope (Ti-E; Nikon).

To visualize the birth scar and bud scars, cells were stained with WGA-Texas Red at the final concentration of $100 \mu \mathrm{g} / \mathrm{ml}$, and $15 \mathrm{z}$ stacks $(0.4 \mu \mathrm{m}$ step $)$ of static images were collected in both GFP and RFP channels, followed by time-lapse imaging only with GFP for mNG-Bud8 (Fig. 2) using a spinning disk confocal microscope (see above). Images of the birth scar and bud scars (Fig. 1E) were also captured similarly but using a Nikon Ti-E microscope fitted with a 100x/1.45 NA PlanApochromat Lambda oil immersion objective lens (Nikon), FITC/GFP, and mCherry/Texas Red filters from Chroma Technology, an Andor iXon Ultra 888 electron-multiplying charge-coupled device (EM CCD) (Andor Technology), and the software NIS Elements (Nikon). Because of the distinct size difference, the birth scar (which is larger than bud scars) can be unambiguously identified from singlecolor imaging with WGA-Texas Red or WGA-FITC.

To make figures for fluorescence images, maximum intensity projections were generated for fluorescence using ImageJ software (National Institutes of Health) for fluorescence images, and single z stack images were used for DIC images. Where indicated, images were deconvolved by the Iterative Constrained Richard-Lucy algorithm using NIS Elements software for figure presentation. Image analyses were performed using ImageJ with summed intensity projections of z stacks after background subtraction. For analysis of Bud8 localization pattern, a fluorescence threshold was set to select localized mNG-Bud8 clusters (as shown in Fig. 2B), and then cells with or without the highlighted pixels at each location were counted (Fig. 2C). To measure the local intensity of mNG-Bud8, the same threshold was applied to all summed intensity projections, and mean intensity was measured among all pixels above the threshold at each location. Each data point in Fig. 2D represents an average of independent imaging sets. For analysis of colocalization of mNG-Bud8 and Rax2-RFP in mother cells, Pearson's correlation coefficient was determined using ImageJ plugin JACoP (Bolte and Cordelières, 2006). To compare the localization of $\mathrm{mNG}$-Bud8 and Rax2-tdTomato in time-lapse images, $\mathrm{mNG}$ Bud8 and Rax2-tdTomato around the cell cortex using ImageJ, freehand lines (3-pixel, $0.432 \mu \mathrm{m}$ wide) were drawn along the cell periphery on single focused z-stack images using ImageJ, and the 
280 fluorescence intensities were then measured along the lines for each time point of time-lapse images

281 (Fig. S1b).

282

\section{Microfluidic imaging, image processing, and image analysis}

Microfluidics setup, growth conditions, and fluorescence microscopy are performed essentially as described in Singh et al. (Singh et al., 2017). Microfluidic devices were fabricated using polydimethylsiloxane (PDMS) by adopting a design used in the Li laboratory (a kind gift from R. Li). Yeast cells suspended at $\sim 10^{6}-10^{7}$ cells $/ \mathrm{ml}$ concentration were slowly loaded onto the device through the inlet until single cells were captured in features at the desired occupancy. Subsequently, fresh synthetic media was applied at the constant flow rate by a syringe pump (Fusion 200; Chemyx). An initial flow rate $(30 \mu \mathrm{l} / \mathrm{min})$ was applied to remove bubbles and wash out nontrapped cells. Thereafter, it was reduced to $10 \mu \mathrm{l} / \mathrm{min}$ to keep trapped mother cells under a positive pressure and to wash out daughter cells consistently. The temperature remained mostly constant at $29 \sim 30^{\circ} \mathrm{C}$ during imaging, and time-lapse images at multiple XY positions were captured ( $5 \mathrm{z}$ stacks, $0.5 \mu \mathrm{m}$ step) by an epifluorescence microscope equipped with a 60x/1.4 NA Plan Apochromat Lambda oil immersion objective lens (Nikon) and DIC (differential interference contrast) optics (Nikon Ti-E; Nikon, Tokyo, Japan). Bright-field and fluorescence images were typically recorded for about $72 \mathrm{hrs}$ at 20-min intervals using NIS Elements (Nikon). Where indicated in figure legends, microfluidic images were deconvolved by the Iterative Constrained Richard-Lucy algorithm (NIS Elements) and cropped at selected ages, preferentially at the cell division or soon after division. Images were adjusted with the same LUTs (look-up tables) setting.

Image analysis was performed after importing nd2 files using ImageJ Bio-Format importer plugin. Fluorescence intensity of PBD-tdTomato was estimated for each cell division cycle by applying the same threshold to the best focused single $\mathrm{z}$ slice using ImageJ. Each cell division was divided into three groups based on the PBD-tdTomato level in the G1 phase (rather than quantifying the exact fluorescence intensity) (Fig. 3): those at the threshold (+/-), middle level (++), and the highest PBD $(+++)$. The highest $(+++)$ and the middle high $(++)$ groups represent the top $7 \%$ and the next $10 \%$ of cell divisions with high PBD, respectively, out of a total of 1,474 cell divisions scored. The majority of the cell divisions displays PBD levels close to the threshold (+/-). A precise quantification of the PBD-tdTomato level on the cell cortex was challenging particularly in the aged cells because of the proximity of the large vacuolar membrane to the plasma membrane. In addition, old bud $8 \Delta$ cells sometimes had PBD-tdTomato accumulated in the vacuolar lumen, which was present very closely to the plasma membrane. It was thus difficult to separate the fluorescence only from the plasma membrane at the resolution of typical microfluidic images. Cell death was marked when it was evident morphologically with abruptly shrinking cell size or cell lysis (from DIC images) and sudden complete loss of normal fluorescence signals or abrupt appearance of strong autofluorescence in the whole cell in both channels. Cell divisions are marked as near senescence or senescence when there was a sudden increase in the cell cycle length $(>5 \sim 6 \mathrm{hrs})$ with no clear evidence of cell death and when no further division was scored within $8 \sim 12$ hrs.

To compare the GFP-Cdc42 level in the strains carrying a single copy (GFP-CDC42) or 8 copies of GFP-Cdc42 (GFP-Cdc42 X 8) on the chromosome, the mean global intensity of GFP in young cells (age 1 2) during cytokinesis or in the G1 phase were quantified from single z slice after background subtraction. The mean intensity of GFP in each cell was plotted in Fig. 4A(b). To quantify the GFPCdc42 level during aging, the mean intensity of GFP was measured similarly at the cytokinesis or G1 phase of each age until death or senescence. Total GFP-Cdc42 level at each age is calculated by multiplying each mean intensity by each cell size and plotted in a different colored line for each cell (Fig. 4B, c). 


\section{Program for RLS measurement}

We developed a Python program for automatic analysis of RLS of multiple single cells in one XY position of capturing. This program allows rapid RLS determination based on fluctuation of a fluorescent marker level during the cell cycle (such as Cdc3-GFP or Cdc3-mCherry marker, whose level drops in the G1 phase when the old ring disassembles). Because this analysis identifies each cell based on its position, the program works well for those cells whose positions within the PDMS pillars have not shifted much during the entire lifespan. RLS measurement of a cell expressing Cdc3-GFP (and PBD-RFP) by the program and ImageJ is compared (see an example in Fig. S2, f). The automatic reading of the Cdc3-GFP level of each capture showed a repeating pattern of a curve, and the number of the valleys on the graph matches the number of cell divisions until death. However, the current program has some limitation because some cells rotate during imaging or shift their positions further away from the PDMS pillars (while these cells remain trapped). The algorithm and the detailed steps of this program are described below.

\section{Cropping the whole chip:}

Since the whole microfluidics chip has a specific layout of chambers (i.e., 4 columns per XY position; and 5 or 6 chambers per column; see Fig. S2, a), this provides a convenient way to crop an XY capture into multiple images covering only one cell in a pair of pillars. Cropping of images can be done by first cutting the whole capture vertically into columns then cutting horizontally. This is done by calculating the average intensity in a small sliding window through the whole image first horizontally and then vertically to determine the cut points (Fig. S2).

Detailed algorithm steps:

1. Load the nd2 image file with BioFormats function from pims library.

2. Keep the DIC channel from the loaded file for cropping.

3. Define a square sliding window with size $1 * \max$ height

4. Collect all the average intensity from $x=0$ to $x=$ max_width

5. Find the max intensity position $p_{-} l$ from average intensities. Add $p_{-} l$ to horizontal cutting points.

6. Set all the average intensity values to 0 and cut one column of chambers out (see Fig. S2, b \& c) if its position $a b s\left(p \_2-p \_l\right)<T$. T is a threshold which should be set slightly larger than the chamber width.

7. Repeat step 5 and 6 for 4 times.

8. Define a square sliding window with size column_width $* 1$

9. Collect all the average intensity from $y=0$ to $y=$ max_width

10. Similar to step 5 and 6 , pick the vertical cutting points and crop each chamber out.

11. Repeat step 10 for 6 times if the column is the first and the third to the left, otherwise 5 times.

12. Return the cropping results.

\section{Statistical analysis}

Data analysis was performed using Prism 8 (GraphPad Software). To determine statistical differences between two sets of data of cell survival, the Gehan-Breslow-Wilcoxon test and Log-rank (MantelCox) test were used. Where indicated in Figure legends, a two-tailed student's test was performed to determine statistical significance: ns (not significant) for $\mathrm{p} \geq 0.05 ;{ }^{*} \mathrm{p}<0.05 ;{ }^{*} \mathrm{p}<0.01 ; * * * \mathrm{p}<$ $0.001 ; * * * * \mathrm{p}<0.0001$. Horizontal lines and error bars on the graphs denote mean $\pm \mathrm{SEM}$. 


\section{Acknowledgments}

We are grateful to R. Li and H. Li for their help with microfluidics, and K. E. Miller for her assistance with microfluidic imaging. We also thank E. Bi, J. R. Pringle, and D. Lew for yeast strains and plasmids. This work has been supported by grants from the National Institutes of Health/National

\section{Supplemental Material}

Figure S1 \& S2

Video S1

Table S1 


\section{REFERENCES}

Altschuler, S.J., Angenent, S.B., Wang, Y., and Wu, L.F. (2008). On the spontaneous emergence of cell polarity. Nature 454, 886-889.

Barton, A.A. (1950). Some aspects of cell division in saccharomyces cerevisiae. J. Gen. Microbiol. 4, 84-86.

Bi, E., and Park, H.-O. (2012). Cell polarization and cytokinesis in budding yeast. Genetics 191, 347387.

Bolte, S., and Cordelières, F.P. (2006). A guided tour into subcellular colocalization analysis in light microscopy. J. Microsc. 224, 213-232.

Chen, K.L., Crane, M.M., and Kaeberlein, M. (2017). Microfluidic technologies for yeast replicative lifespan studies. Mech. Ageing Dev. 161, 262-269.

Chen, T., Hiroko, T., Chaudhuri, A., Inose, F., Lord, M., Tanaka, S., Chant, J., and Fujita, A. (2000). Multigenerational cortical inheritance of the Rax2 protein in orienting polarity and division in yeast. Science 290, 1975-1978.

Egilmez, N.K., and Jazwinski, S.M. (1989). Evidence for the involvement of a cytoplasmic factor in the aging of the yeast Saccharomyces cerevisiae. J. Bacteriol. 171, 37-42.

Florian, M.C., Dorr, K., Niebel, A., Daria, D., Schrezenmeier, H., Rojewski, M., Filippi, M.D., Hasenberg, A., Gunzer, M., Scharffetter-Kochanek, K., Zheng, Y., and Geiger, H. (2012). Cdc42 activity regulates hematopoietic stem cell aging and rejuvenation. Cell Stem Cell 10, 520-530.

Florian, M.C., Klose, M., Sacma, M., Jablanovic, J., Knudson, L., Nattamai, K.J., Marka, G., Vollmer, A., Soller, K., Sakk, V., Cabezas-Wallscheid, N., Zheng, Y., Mulaw, M.A., Glauche, I., and Geiger, H. (2018). Aging alters the epigenetic asymmetry of HSC division. PLoS biology 16, e2003389.

Gaillard, J.M., and Lemaître, J.F. (2017). The Williams' legacy: A critical reappraisal of his nine predictions about the evolution of senescence. Evolution 71, 2768-2785.

Giebel, B. (2008). Cell polarity and asymmetric cell division within human hematopoietic stem and progenitor cells. Cells Tissues Organs 188, 116-126.

Guthrie, C., and Fink, G.R. (1991). Guide to Yeast Genetics and Molecular Biology. Academic Press: San Diego.

Harkins, H.A., Pagé, N., Schenkman, L.R., De Virgilio, C., Shaw, S., Bussey, H., and Pringle, J.R. (2001). Bud8p and Bud9p, proteins that may mark the sites for bipolar budding in yeast. Mol. Biol. Cell 12, 2497-2518.

Jazwinski, S.M. (2001). New clues to old yeast. Mech. Ageing Dev. 122, 865-882.

Jazwinski, S.M., Kim, S., Lai, C.Y., and Benguria, A. (1998). Epigenetic stratification: the role of individual change in the biological aging process. Exp. Gerontol. 33, 571-580.

Johnston, J.R. (1966). Reproductive capacity and mode of death of yeast cells. Antonie Van Leeuwenhoek 32, 94-98.

Kang, P.J., Angerman, E., Nakashima, K., Pringle, J.R., and Park, H.-O. (2004). Interactions among Rax1p, Rax2p, Bud8p, and Bud9p in marking cortical sites for bipolar bud-site selection in yeast. Mol. Biol. Cell 15, 5145-5157. 
Kang, P.J., Lee, M.E., and Park, H.-O. (2014). Bud3 activates Cdc42 to establish a proper growth site in budding yeast. J. Cell Biol. 206, 19-28.

Kang, P.J., Miller, K.E., Guegueniat, J., Beven, L., and Park, H.-O. (2018). The shared role of the Rsr1 GTPase and Gic1/Gic2 in Cdc42 polarization. Mol. Biol. Cell 29, 2359-2369.

Kennedy, B.K., Austriaco, N.R., Jr., and Guarente, L. (1994). Daughter cells of Saccharomyces cerevisiae from old mothers display a reduced life span. J. Cell Biol. 127, 1985-1993.

Kenyon, C. (2001). A conserved regulatory system for aging. Cell 105, 165-168.

Lo, W.C., Lee, M.E., Narayan, M., Chou, C.S., and Park, H.-O. (2013). Polarization of diploid daughter cells directed by spatial cues and GTP hydrolysis of Cdc42 budding yeast. PLoS One 8 , e56665.

McCormick, M.A., Delaney, J.R., Tsuchiya, M., Tsuchiyama, S., Shemorry, A., Sim, S., Chou, A.C., Ahmed, U., Carr, D., Murakami, C.J., Schleit, J., Sutphin, G.L., Wasko, B.M., Bennett, C.F., Wang, A.M., Olsen, B., Beyer, R.P., Bammler, T.K., Prunkard, D., Johnson, S.C., Pennypacker, J.K., An, E., Anies, A., Castanza, A.S., Choi, E., Dang, N., Enerio, S., Fletcher, M., Fox, L., Goswami, S., Higgins, S.A., Holmberg, M.A., Hu, D., Hui, J., Jelic, M., Jeong, K.S., Johnston, E., Kerr, E.O., Kim, J., Kim, D., Kirkland, K., Klum, S., Kotireddy, S., Liao, E., Lim, M., Lin, M.S., Lo, W.C., Lockshon, D., Miller, H.A., Moller, R.M., Muller, B., Oakes, J., Pak, D.N., Peng, Z.J., Pham, K.M., Pollard, T.G., Pradeep, P., Pruett, D., Rai, D., Robison, B., Rodriguez, A.A., Ros, B., Sage, M., Singh, M.K., Smith, E.D., Snead, K., Solanky, A., Spector, B.L., Steffen, K.K., Tchao, B.N., Ting, M.K., Vander Wende, H., Wang, D., Welton, K.L., Westman, E.A., Brem, R.B., Liu, X.G., Suh, Y., Zhou, Z., Kaeberlein, M., and Kennedy, B.K. (2015). A Comprehensive Analysis of Replicative Lifespan in 4,698 Single-Gene Deletion Strains Uncovers Conserved Mechanisms of Aging. Cell Metab. 22, 895-906.

Meitinger, F., Khmelinskii, A., Morlot, S., Kurtulmus, B., Palani, S., Andres-Pons, A., Hub, B., Knop, M., Charvin, G., and Pereira, G. (2014). A memory system of negative polarity cues prevents replicative aging. Cell 159, 1056-1069.

Miller, K.E., Lo, W.C., Chou, C.S., and Park, H.O. (2019). Temporal regulation of cell polarity via the interaction of the Ras GTPase Rsr1 and the scaffold protein Bem1. Mol. Biol. Cell 30, 2543-2557.

Miller, K.E., Lo, W.C., Lee, M.E., Kang, P.J., and Park, H.-O. (2017). Fine-tuning the orientation of the polarity axis by Rga1, a Cdc42 GTPase-activating protein. Mol. Biol. Cell 28, 3773-3788.

Mortimer, R.K., and Johnston, J.R. (1959). Life span of individual yeast cells. Nature 183, 1751-1752.

Nestelbacher, R., Laun, P., and Breitenbach, M. (1999). Images in experimental gerontology. A senescent yeast mother cell. Exp. Gerontol. 34, 895-896.

Okada, S., Leda, M., Hanna, J., Savage, N.S., Bi, E., and Goryachev, A.B. (2013). Daughter cell identity emerges from the interplay of Cdc42, septins, and exocytosis. Dev. Cell 26, 148-161.

Okada, S., Lee, M.E., Bi, E., and Park, H.-O. (2017). Probing Cdc42 Polarization Dynamics in Budding Yeast Using a Biosensor. Methods Enzymol. 589, 171-190.

Singh, P., Ramachandran, S.K., Zhu, J., Kim, B.C., Biswas, D., Ha, T., Iglesias, P.A., and Li, R. (2017). Sphingolipids facilitate age asymmetry of membrane proteins in dividing yeast cells. Mol.

Biol. Cell 28, 2712-2722. 
Smith, G.R., Givan, S.A., Cullen, P., and Sprague, G.F., Jr. (2002). GTPase-activating proteins for Cdc42. Eukaryotic Cell 1, 469-480.

Steffen, K.K., Kennedy, B.K., and Kaeberlein, M. (2009). Measuring replicative life span in the budding yeast. J Vis Exp.

Steinkraus, K.A., Kaeberlein, M., and Kennedy, B.K. (2008). Replicative aging in yeast: the means to the end. Annual review of cell and developmental biology 24, 29-54.

Taheri, N., Köhler, T., Braus, G.H., and Mösch, H.-U. (2000). Asymmetrically localized Bud8p and Bud9p proteins control yeast cell polarity and development. The EMBO J. 19, 6686-6696.

Tong, Z., Gao, X.D., Howell, A.S., Bose, I., Lew, D.J., and Bi, E. (2007). Adjacent positioning of cellular structures enabled by a Cdc42 GTPase-activating protein-mediated zone of inhibition. J. Cell Biol. 179, 1375-1384.

Wang, L., Yang, L., Debidda, M., Witte, D., and Zheng, Y. (2007). Cdc42 GTPase-activating protein deficiency promotes genomic instability and premature aging-like phenotypes. Proceedings of the National Academy of Sciences of the United States of America 104, 1248-1253.

Wasko, B.M., and Kaeberlein, M. (2014). Yeast replicative aging: a paradigm for defining conserved longevity interventions. FEMS Yeast Res 14, 148-159.

Williams, G.C. (1957). Pleiotropy, natural selection, and the evolution of senescence. Evolution 11, 398-411.

Wu, C.F., Chiou, J.G., Minakova, M., Woods, B., Tsygankov, D., Zyla, T.R., Savage, N.S., Elston, T.C., and Lew, D.J. (2015). Role of competition between polarity sites in establishing a unique front. eLife 4, 11611.

Xie, Z., Zhang, Y., Zou, K., Brandman, O., Luo, C., Ouyang, Q., and Li, H. (2012). Molecular phenotyping of aging in single yeast cells using a novel microfluidic device. Aging Cell 11, 599-606.

Zahner, J.E., Harkins, H.A., and Pringle, J.R. (1996). Genetic analysis of the bipolar pattern of bud-site selection in the yeast Saccharomyces cerevisiae. Mol. Cell. Biol. 16, 1857-1870. 


\section{FIGURE LEGENDS}

\section{Figure 1. RLS measurement by micromanipulation}

Graphs show the percentage of surviving cells after the indicated number of divisions. All strains are in the same background (see Table S1). The median survival age of each strain is indicated in parentheses. $P$ values are obtained from Log-rank (Mantel-Cox) test.

A. WT $(n=28), \operatorname{rax} 1 \Delta(n=25)$, and $\operatorname{rax} 2 \Delta(n=25)$ strains. $p=0.0039$ (WT vs $\operatorname{rax} 1 \Delta), p=0.0070$ (WT vs rax2 $\Delta$ ).

B. WT $(n=26)$, bem $3 \Delta(n=20)$, and rga2 $\Delta(n=24)$ strains. $\mathrm{p}<0.0001$ (WT vs bem $3 \Delta$; and WT vs $\operatorname{rga} 2 \Delta)$.

C. WT $(n=28), r s r 1 \Delta(n=48), \operatorname{axl} 1 \Delta(n=26)$, and $\operatorname{axl} 2 \Delta(n=31)$ strains. $p=0.84($ WT vs $r s r 1 \Delta)$, 0.97 (WT vs $\operatorname{axll\Delta } \Delta)$, and 0.89 (WT vs $\operatorname{axl} 2 \Delta)$.

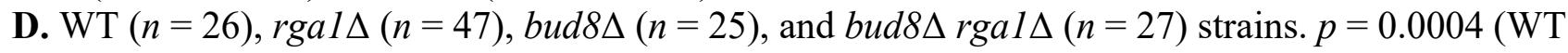

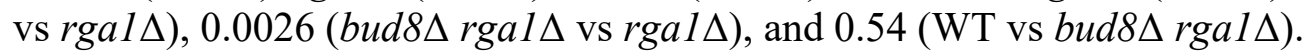

E. WGA-Texas Red staining of WT and bud8 $\Delta$ rgal $\Delta$ cells. Bud scars (marked with arrows) are usually smaller and show stronger fluorescence than the birth scar (marked with arrowheads).

Fluorescence images were deconvolved. Scale bar: $5 \mu \mathrm{m}$.

\section{Figure 2. Bud8 localizes to CRMs and the distal pole of mother cells}

A. Localization of mNG-Bud8 in haploid WT, rax $1 \Delta$, and rgal $\Delta$ cells. New mother cells with their first bud are shown. Pink arrows mark Bud8 localized to the proximal poles; blue arrows and arrowheads mark Bud8 at the distal pole of mother and bud, respectively. Note: Budding occurs within the birth scar of rax $1 \Delta$ and $\operatorname{rgal} \Delta$ cells unlike axially budding WT haploids. Scale bar: $3 \mu \mathrm{m}$. See also Fig. S1a.

B. Bud8 localized in multiple clusters in a mother cell that had budded several times. These Bud8 signals overlap with bud scars (white arrows) and the birth scar (pink arrow). The mNG-Bud8 intensity above threshold (shown) is considered as localized signals. Scale bar: $3 \mu \mathrm{m}$.

C. The percentage of cells with localized Bud8 (see legend to Fig. 2B) at marked locations is shown for each strain. Each symbol shows the mean value of an independent imaging set $(\mathrm{n}>120$ cells for each strain). Horizontal lines and error bars denote mean $\pm \mathrm{SEM}$; and $p$ values are shown from unpaired two-tailed t-test: ns (not significant) for $p \geq 0.05, * p<0.05, * * * * * p<0.00001$ and $* * * * * * p<$ 0.000001 .

D. The mNG-Bud8 localized to the distal pole of mother cells and buds is quantified from the same image sets analyzed in Fig. 2C (see legend to Fig. 2C).

E. Colocalization of mNG-Bud8 and Rax2-RFP in a mother cell. Pearson's correlation coefficient $r$ (in mother cells) $=0.72$. See Fig. S1, b.

Figure 3. Cdc42-GTP is sporadically elevated in WT cells but rarely in bud8 $\Delta$ cells.

A \& B. Single-cell trajectories (each row) are aligned with the last cell division of (A) WT and (B) bud $8 \Delta$ cells $(n=40$ for each strain). Each box shows the PBD-RFP level (threshold $+/$ - or higher) in the G1 phase of each cell cycle. A black box denotes cell death. Divisions near senescence (green boxes) are scored as a sudden increase in the cell cycle length ( $>5 \mathrm{hrs}$ ) with no clear sign of cell death 
and are further distinguished with the PBD level as marked. Ages are shown relative to the last cell division.

C. Images of a WT cell with representative PBD levels during the cytokinesis or G1 phase are shown. The initially loaded cell (at age -28) is shown with an arrow marking the direction of media flow. Scale bar: $3 \mu \mathrm{m}$. See Video S1.

D. The number of cell divisions with the PBD level higher than the threshold (+/-) are compared between WT and bud8 $\Delta$ cells. Total numbers of cell divisions scored: $n=679$ (WT) and $n=795$

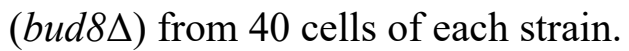

\section{Figure 4. Overexpression of $\mathrm{Cdc} 42$ causes premature aging}

A (a). The level of GFP-Cdc42 in young cells (age 1) of the GFP-CDC42 and GFP-CDC42ov strains. Dots represent mean global intensity of GFP-Cdc42 in individual cells $(\mathrm{n}=21, G F P-C D C 42 ; \mathrm{n}=39$, $C D C 42 o v)$. Horizontal lines and error bars denote mean $\pm \mathrm{SEM}$, and $p$ values are shown from unpaired two-tailed t-test.

A (b). RLS estimation by microfluidic imaging at $29^{\circ} \mathrm{C}$. WT $(n=70)$ carries the endogenous $C D C 42$, and the $C D C 42$ ov strain $(\mathrm{n}=90)$ carries GFP-CDC42 X 8 on the chromosome. Graph shows the percentage of surviving cells after the indicated number of cell divisions (mean $\pm \mathrm{SEM}$ ). The median number of cell divisions that each strain has undergone before senescence or death is indicated in parentheses. $p<0.0001$ (WT vs CDC42ov) from Log-rank (Mantel-Cox) test. Note: The two strains are in the same background that is different from the one used in Fig. 1 (see Table S1).

B. Microfluidic images of GFP-CDC42ov (also expresses Cdc3-mCherry) are shown at selected time points. Numbers at the top denote ages, and the numbers at the bottom indicate the relative time (hr: $\mathrm{min}$ ) from the first image at age 1. Fluorescent images were deconvolved. Scale bars: $5 \mu \mathrm{m}$. (a) A representative cell showing characteristics of premature aging and elevation of GFP-Cdc42 near senescence. White arrows mark a mother cell, which has likely entered senescence at age 6 7. Blue arrows mark a bud from the 7th daughter cell, which died at its first cell division. Daughter cells are larger than mother at the 6th and 7th divisions. (b) A representative cell with a longer RLS than the average undergoes normal asymmetric cell divisions until death at age 17. (c) Total intensity of GFP$\mathrm{Cdc} 42$ during cytokinesis $\sim \mathrm{G} 1$ is plotted at each age of GFP-CDC42ov cells. Colored lines represent individual mother cells from age 1 to death or senescence. Red and blue lines with markers represent the cells shown in (a) and (b), respectively.

Figure 5. A model. In newborn daughter cells and young mother cells, negative polarity factors such as Rga1, which is recruited to CRMs via Nis1 and Nba1, inhibit Cdc42 activity at the cell cortex. Bud8, which localizes to CRMs and the distal pole, may counteract negative polarity signaling in older cells, which have more CRMs, leading to an elevation of Cdc42 activity at the overall cell cortex. The Nis1-Nba1 complex is omitted in older cells for simplicity. 


\section{LEGENDS FOR SUPPLEMENTARY FIGURES AND VIDEO}

\section{Figure S1. Time-lapse images of mNG-Bud8 and Rax2-tdTomato}

a. Localization of mNG-Bud8 in a haploid WT cell, which is pre-stained with WGA-Texas Red, at $30^{\circ} \mathrm{C}$. Blue arrows and arrowheads mark the distal pole of mother and bud (which became a newborn daughter cell), respectively. Pink arrowheads mark Bud8 signal at the proximal pole of daughter cell. White arrow and arrowheads mark new buds formed from the initial mother and daughter cells, respectively. Scale bar: $5 \mu \mathrm{m}$.

b. Time-lapse images of mNG-Bud8 and Rax2-tdTomato. Blue arrowheads mark distal pole of a bud (and a newborn daughter cell). A white arrow and a white arrowhead mark new bud emergence in mother and daughter cell, respectively. Colocalization of Bud8 and Rax 2 in mother cells appears in multiple puncta at CRMs. Plots of normalized fluorescence intensity profiles of Bud8 and Rax 2 along the periphery of the mother cell at selected timepoints (marked with yellow arrows) are shown below. Scale bar: $5 \mu \mathrm{m}$.

\section{Figure S2. A Python program and RLS estimation}

To crop the whole image (a), first, a window (red box) moving horizontally (blue arrow) is used. The average pixel intensity for the window is shown in (b). Cutting between peaks in (b) generates image slices shown in (c). Next, sliding a window (red box in c) vertically generates the average intensity shown in (d). Cutting between peaks as marked in (d) results in a single cell image (e) and the intensity values of each fluorescence channel. A cell (expressing PBD-tdTomato and Cdc3-GFP) loaded in a single trap is shown in (e), and the highest pixel intensity of Cde3-GFP in a WT cell is quantified at each time point by either the Python program or ImageJ. The number of valleys of this graph represents the number of cell divisions that this cell has undergone until death.

\section{Video S1.}

Microfluidic images of a haploid WT cell expressing Gic2-PBD-RFP and Cdc3-GFP (overlayed with DIC). Images were captured every 20 min using a widefield microscope (Nikon Ti-E; Nikon) at $29 \sim 30^{\circ} \mathrm{C}$. The video shows frames for $50 \mathrm{hrs}$ starting from the initial loading until death. Display rate is 8 frames/s. Selected images are shown in Fig. 3C. 
Table SI. Yeast strains used in this study

\begin{tabular}{|c|c|c|}
\hline Strain & Relevant Genotype $^{\mathrm{a}}$ & Source/Comments \\
\hline BY4741@ & MATa his3- $\Delta 1$ leu2 $\Delta 0$ met $15 \Delta 0$ ura3 $\Delta 0$ & Open Biosystems \\
\hline HPY2206@ & $M A T \mathbf{a} \operatorname{rgal} \triangle: \because K A N$ & Open Biosystems \\
\hline HPY2438@ & $M A T \mathbf{a}$ bud $8 \Delta: \because K A N$ & Open Biosystems \\
\hline HPY3700@ & MATa bud8 $\triangle:: K A N$ rgal $\triangle:: K A N$ & This study \\
\hline HPY2247@ & $M A T \mathbf{a} \operatorname{rga} 2 \Delta: \because K A N$ & Open Biosystems \\
\hline HPY2425@ & $M A T \mathbf{a}$ bem3 $\triangle: K A N$ & Open Biosystems \\
\hline HPY664@ & $M A T \mathbf{a} \operatorname{rsr} 1 \triangle \because K A N$ & Open Biosystems \\
\hline HPY1187@ & $M A T \mathbf{a} \operatorname{axl} 1 \triangle: \because K A N$ & Open Biosystems \\
\hline HPY1444@ & $M A T \mathbf{a} \operatorname{axl} \triangle \triangle: \because K A N$ & Open Biosystems \\
\hline HPY2325 & MATa GIC2-PBD-tdTomato-URA3 CDC3-GFP-LEU2 & This study ${ }^{b, c}$ \\
\hline HPY3469 & $\begin{array}{l}\text { MATa bud8A::TRP1 GIC2-PBD-tdTomato-URA3 CDC3-GFP- } \\
\text { LEU2 }\end{array}$ & This study \\
\hline HPY3586 & $M A T \mathbf{a}$ bud8A::TRP1 ymNG-BUD8-URA3 & This study ${ }^{\mathrm{d}}$ \\
\hline HPY3592 & $\begin{array}{l}M A T \mathbf{a} / M A T \alpha \text { bud8A::TRP1/ bud8A::TRP1 ymNG-BUD8-URA3/ } \\
\text { ymNG-BUD8-URA3 }\end{array}$ & This study \\
\hline HPY3671 & $M A T \mathbf{a}$ bud8A::TRP1 ymNG-BUD8-URA3 $\operatorname{rax} 1 \triangle:: H I S 3$ & This study \\
\hline HPY3672\# & MATa bud8A::TRP1 ymNG-BUD8-URA3 rgal $\triangle:: H I S 3$ & This study \\
\hline HPY3702 & MATa bud8A::TRP1 ymNG-BUD8-URA3 RAX2-tdTomato-KAN & This study \\
\hline HPY3713 & $\begin{array}{l}\text { MATa bud8A:: TRP1 rgal } \triangle:: \text { HIS3 GFP-RGA1-URA3 RAX2- } \\
\text { tdTomato-KAN }\end{array}$ & This study \\
\hline HPY3721 & $\begin{array}{l}\text { MATa } c d c 42: \because T R P 1 \text { GFP-CDC42(8X)-URA3 CDC3-mCherry- } \\
\text { LEU2 }\end{array}$ & This study \\
\hline HPY3733 & $\begin{array}{l}\text { MATa } c d c 42 \triangle: \because T R P 1 \text { GFP-CDC42(1X)-URA3 CDC3-mCherry- } \\
\text { LEU2 }\end{array}$ & This study ${ }^{\mathrm{f}}$ \\
\hline
\end{tabular}

a Strains marked with * are derived from YEF473 [1]; strains marked with @ are derived BY4741 (in S288C background).

b YIplac128-CDC3-GFP (a gift from E. Bi) [2] was linearized with BglII and integrated into the CDC3 locus. c YIp211-GIC2-PBD-tdTomato carrying Gic2(1-208)-tdTomato (a gift from E. Bi) [2] was linearized with ApaI and integrated into the URA3 locus.

d YIp211-ymNG-BUD8 (pHP2274) was linearized by digesting with BamHI and then integrated into the $B U D 8$ locus. To fuse ymNG (mNeonGreen) to the N-terminus of Bud8, YIp211-ymNG-BUD8 was generated by replacing the HA epitope sequence of YIp211-HA-BUD8 (a gift from J. Pringle) with the NotI cassette carrying the ymNG coding sequence derived from pFA6a-ymNeonGreen-CaURA3 (Addgene \#125703). 
e Derived from DLY13891 (a gift from D. Lew)[3].

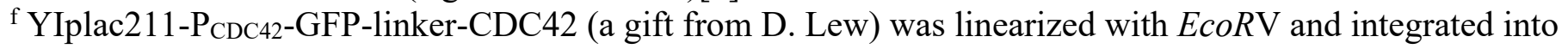
the URA3 locus.

\section{References for Supplemental Table S1}

1. Bi E, Pringle JR. ZDS1 and ZDS2, genes whose products may regulate Cdc42p in Saccharomyces cerevisiae. Mol Cell Biol. 1996;16:5264-75.

2. Tong Z, Gao XD, Howell AS, Bose I, Lew DJ, Bi E. Adjacent positioning of cellular structures enabled by a Cdc42 GTPase-activating protein-mediated zone of inhibition. J Cell Biol. 2007;179(7):1375-84. doi: 10.1083/jcb.200705160. PubMed PMID: PMID: 18166650; PubMed Central PMCID: PMCPMC2373499.

3. Wu CF, Chiou JG, Minakova M, Woods B, Tsygankov D, Zyla TR, et al. Role of competition between polarity sites in establishing a unique front. eLife. 2015;4:11611. Epub 2015/11/03 06:00. PubMed PMID: 26523396. 
A

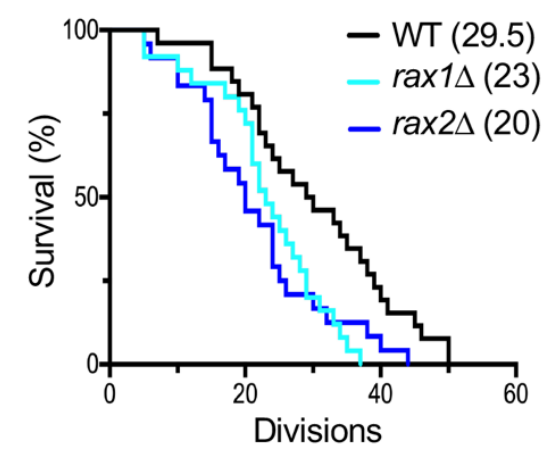

D

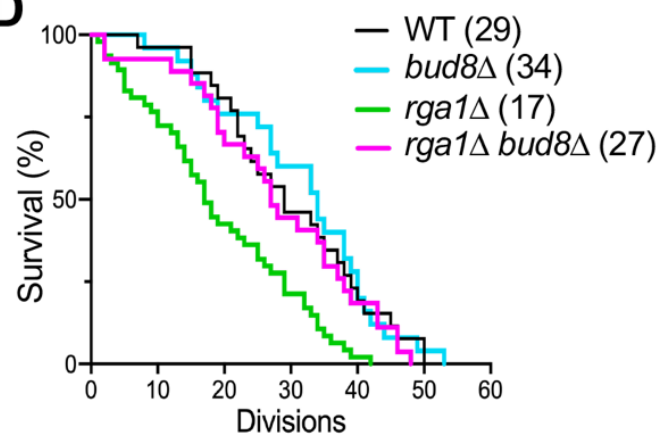

B

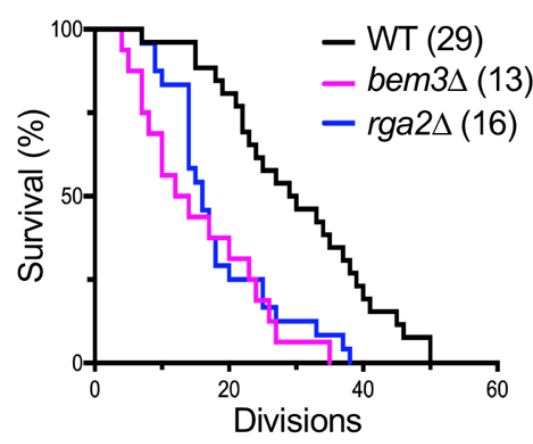

$\mathbf{E}$

E WT

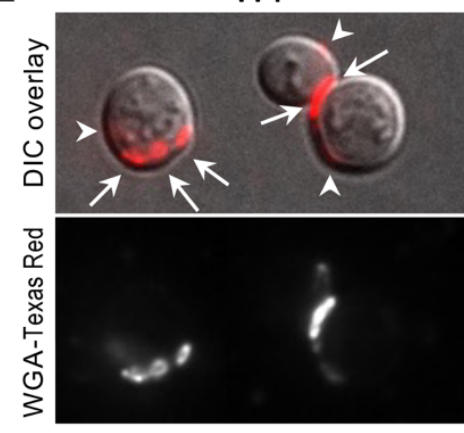

C
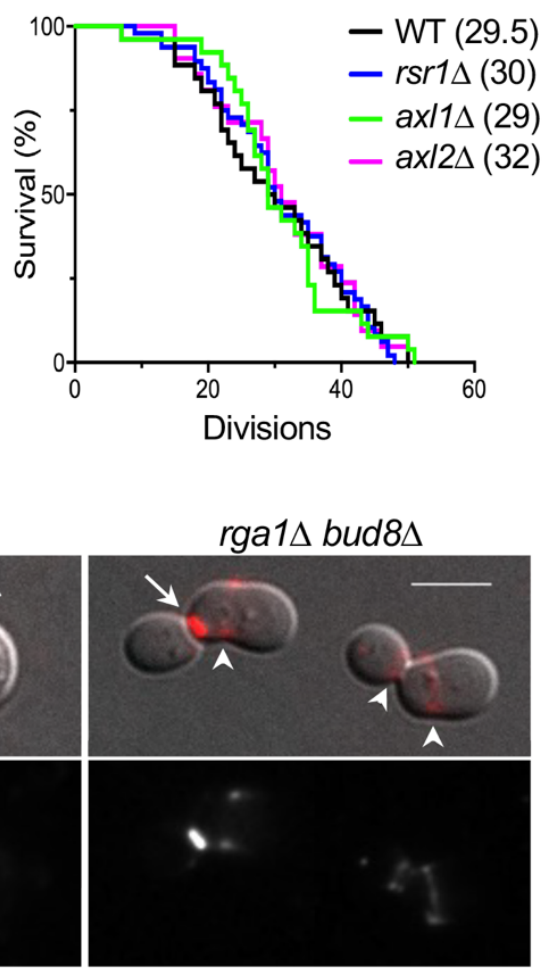

Figure 1. RLS measurement by micromanipulation

Graphs show the percentage of surviving cells after the indicated number of divisions. All strains are in the same background (see Table S1). The median survival age of each strain is indicated in parentheses. $P$ values are obtained from Log-rank (Mantel-Cox) test.

A. WT (black, $n=28$ ), $\operatorname{rax} 1 \Delta$ (light blue, $n=25$ ), and $\operatorname{rax} 2 \Delta$ (blue, $n=25$ ) strains. $p=0.0039$ (WT vs $\operatorname{rax} 1 \Delta$ ), $p=0.0070$ (WT vs $\operatorname{rax} 2 \Delta$ ).

B. WT (black, $n=26$ ), bem $3 \Delta$ (magenta, $n=20$ ), and $\operatorname{rga} 2 \Delta$ (blue, $n=24$ ) strains. $\mathrm{p}<0.0001$ (WT vs bem $3 \Delta$ and WT vs rga $2 \Delta)$.

C. WT (black, $n=28$ ), $r s r l \Delta$ (blue, $n=48$ ), $\operatorname{axl} 1 \Delta$ (green, $n=26$ ), and $\operatorname{axl} 2 \Delta$ (magenta, $n=31$ ) strains. $p=0.84$ (WT vs $r s r l \Delta$ ), $p=0.97$ (WT vs $\operatorname{axl} 1 \Delta$ ), $p=0.89$ (WT vs $\operatorname{axl} 2 \Delta$ ).

D. WT (black, $n=26$ ), rgal $\Delta$ (green, $n=47$ ), bud8 $($ light blue, $n=25$ ), and bud $8 \Delta \operatorname{rgal} \Delta$ (magenta, $n=27$ ) strains. $p=0.0004$ (WT vs $\operatorname{rgal} \Delta$ ), $p=0.0026$ (bud8 $\Delta$ rgal $\Delta$ vs $\operatorname{rgal} \Delta$ ), $p=0.54$

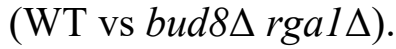

E. WGA-Texas Red staining of WT and bud8 $\Delta$ rgal $\Delta$ cells. Bud scars (marked with arrows) are usually smaller and show stronger fluorescence signals than the birth scar (marked with arrowheads). Fluorescence images were deconvolved. Scale bar: $5 \mu \mathrm{m}$. 
A

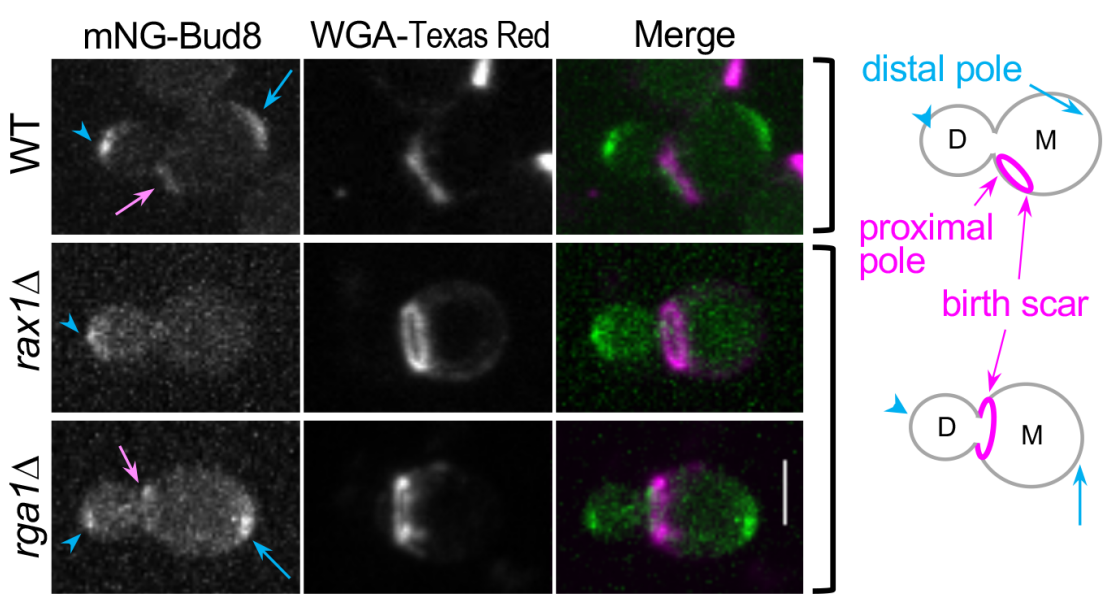

B

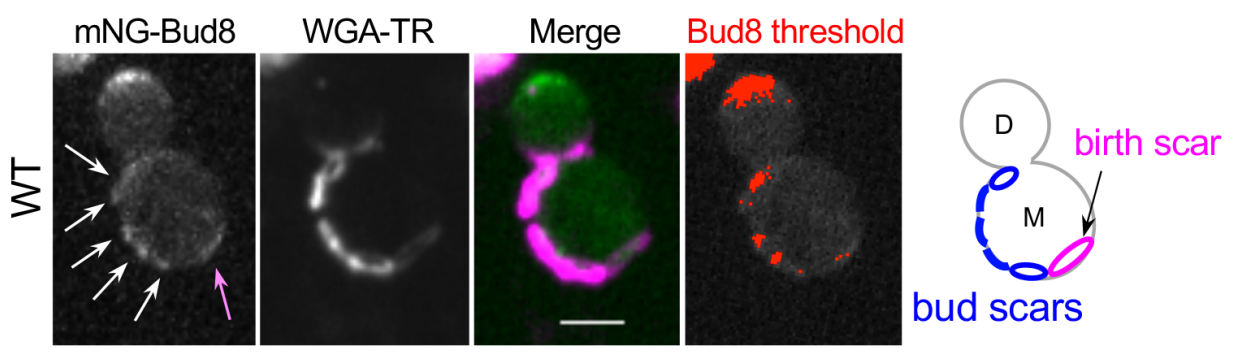

C
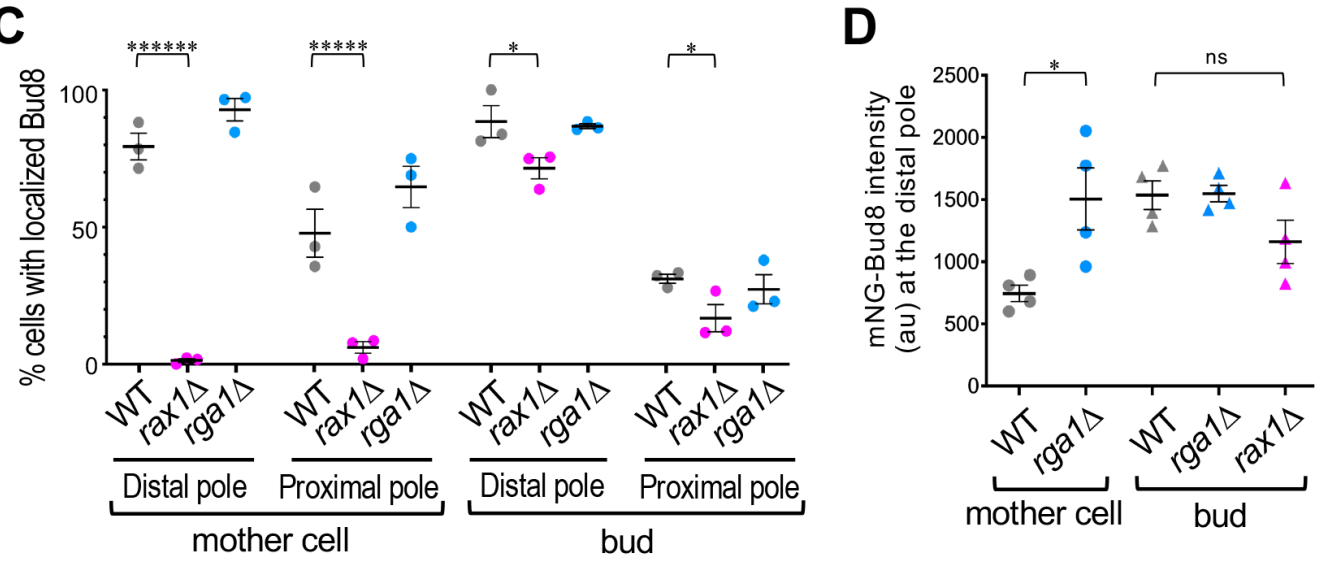

E

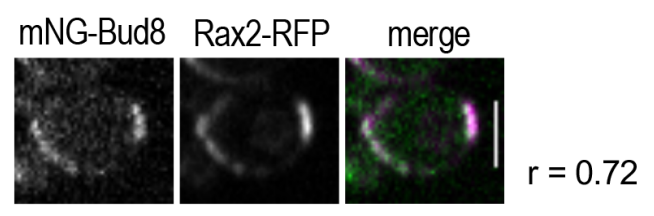

Figure 2. Bud8 localizes to CRMs and the distal pole of mother cells

A. Localization of mNG-Bud8 in haploid WT, $\operatorname{rax} 1 \Delta$, and $\operatorname{rgal} \Delta$ cells. New mother cells with their first bud are shown, as indicated by the birth scar but no bud scar stained with WGA-Texas Red in these cells. Pink arrows mark Bud8 localized to the proximal poles; blue arrows and arrowheads mark Bud8 at the distal pole of mother and bud, respectively. Scheme on the right highlights budding within the birth scar of $\operatorname{rax} 1 \Delta$ and $\operatorname{rgal} \Delta$ cells unlike WT, which buds in the axial pattern. Scale bar: $3 \mu \mathrm{m}$. See also Fig. S1a.

B. Bud8 localized in multiple clusters in a mother cell that had budded several times. These Bud8 signals overlap with bud scars (white arrows) and the birth scar (pink arrow). The mNG-Bud8 intensity above threshold (shown) is considered as localized signals. Scale bar: $3 \mu \mathrm{m}$. 
C. The percentage of cells with localized Bud8 (above the threshold shown in Fig. 2B) at marked locations are shown for each strain. Each symbol shows the mean value of an independent imaging set ( $\mathrm{n}>120$ cells for each sample). Lines denote mean (horizontal lines) $\pm \mathrm{SEM}$ (error bars); and $p$ values are shown from unpaired two-tailed t-test: ns (not significant) for $p \geq 0.05,{ }^{*} p<0.05, * * * * * p<$ 0.00001 and $* * * * * * p<0.000001$.

D. The intensity of mNG-Bud8 localized to the distal pole of mother cells and buds is quantified from the same image sets analyzed in Fig. 2C (see legend to Fig. 2C).

E. Colocalization analysis of $\mathrm{mNG}-\mathrm{Bud} 8$ and Rax2-RFP. Pearson's correlation coefficient $\mathrm{r}$ (in mother cells) $=0.72$. See Fig. S1b. 
A

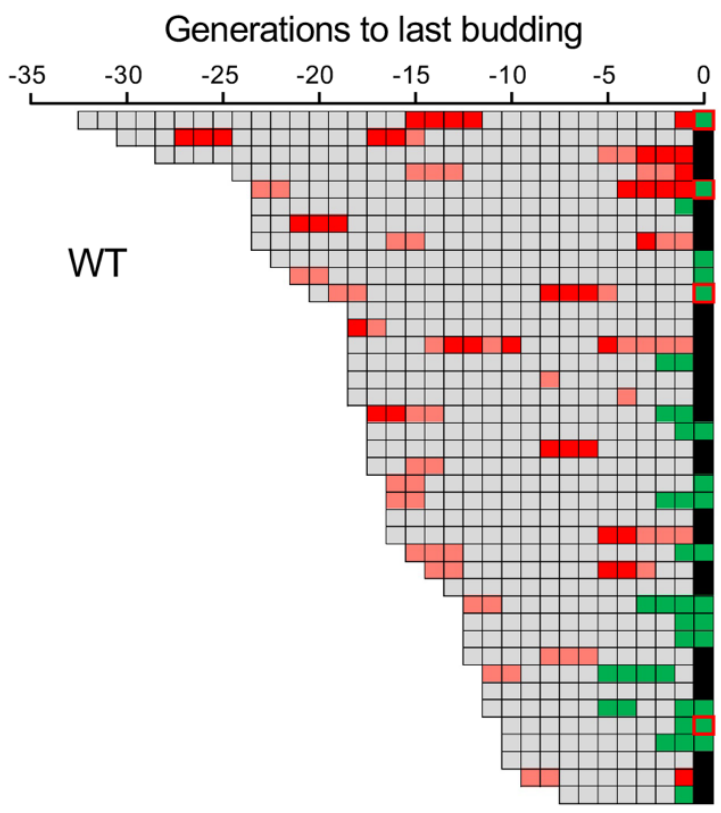

C

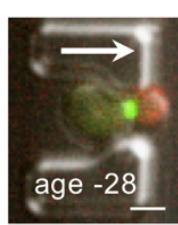

$$
\text { [PBD] level }
$$$$
+/-+++++
$$$$
\square \square \square \text { Normal division timing }
$$$$
\square \quad \square \text { (near) senescence }
$$
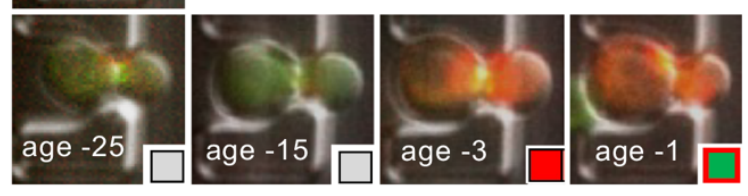

B
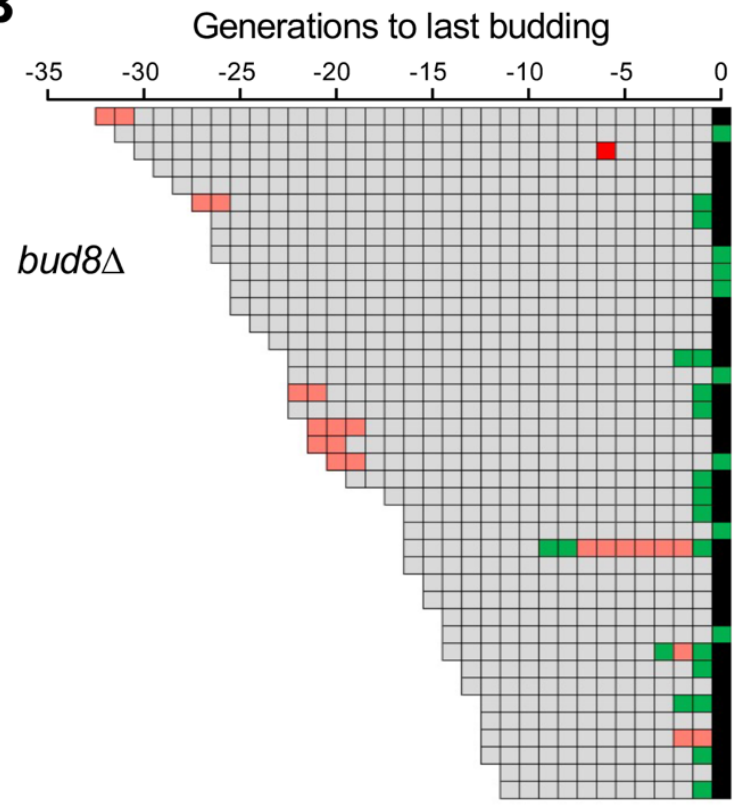

D

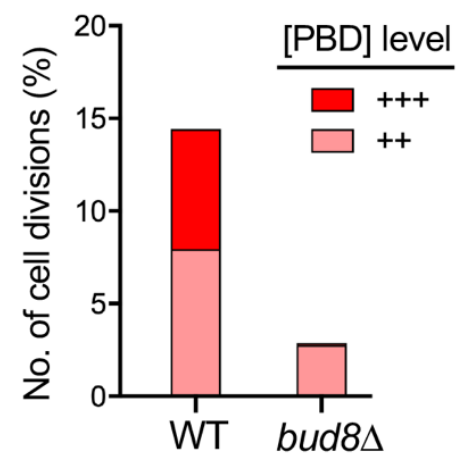

Figure 3. Cdc42-GTP is sporadically elevated in WT cells but rarely in bud8 $\Delta$ cells.

A \& B. Single-cell trajectories (each row) are aligned with the last cell division of each cell of (A) WT and (B) bud8 8 . Each box shows the PBD-RFP level in the G1 phase of each cell cycle: threshold (+/-) (gray), medium high (light red), and high (red). $n=40$ for each strain. A black box denotes cell death. Divisions near senescence (green boxes) are scored as a sudden increase in the cell cycle length $(>5$ hrs) with no clear sign of cell death and are further distinguished with the PBD level as marked. Ages are shown relative to the last cell division.

C. Images of a WT cell with representative PBD levels during the cytokinesis or G1 phase are shown. The initially loaded cell (at age -28) is shown with an arrow marking the direction of media flow. Scale bar: $3 \mu \mathrm{m}$. See Video S1.

D. The number of cell divisions with the PBD level higher than the threshold (+/-) are compared between WT and bud8 $\Delta$ cells. Total numbers of cell divisions scored: $\mathrm{n}=679$ (WT) and $\mathrm{n}=795$

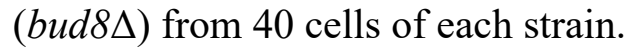


A

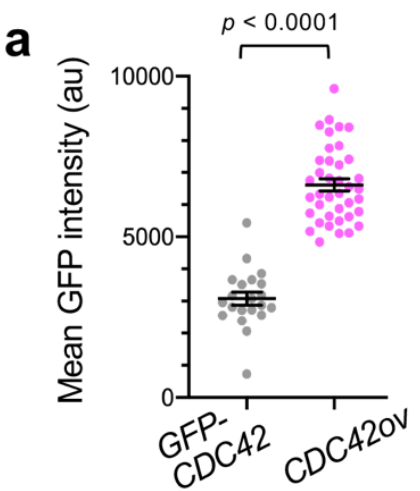

b

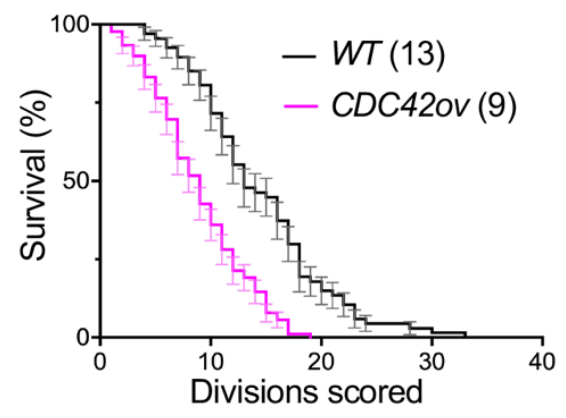

B

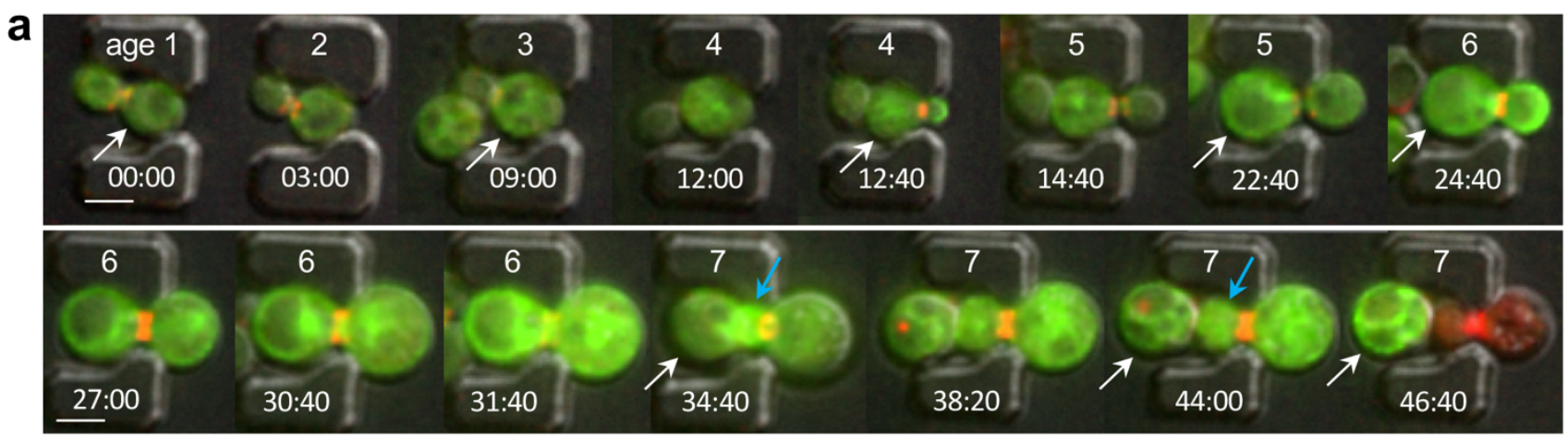

b

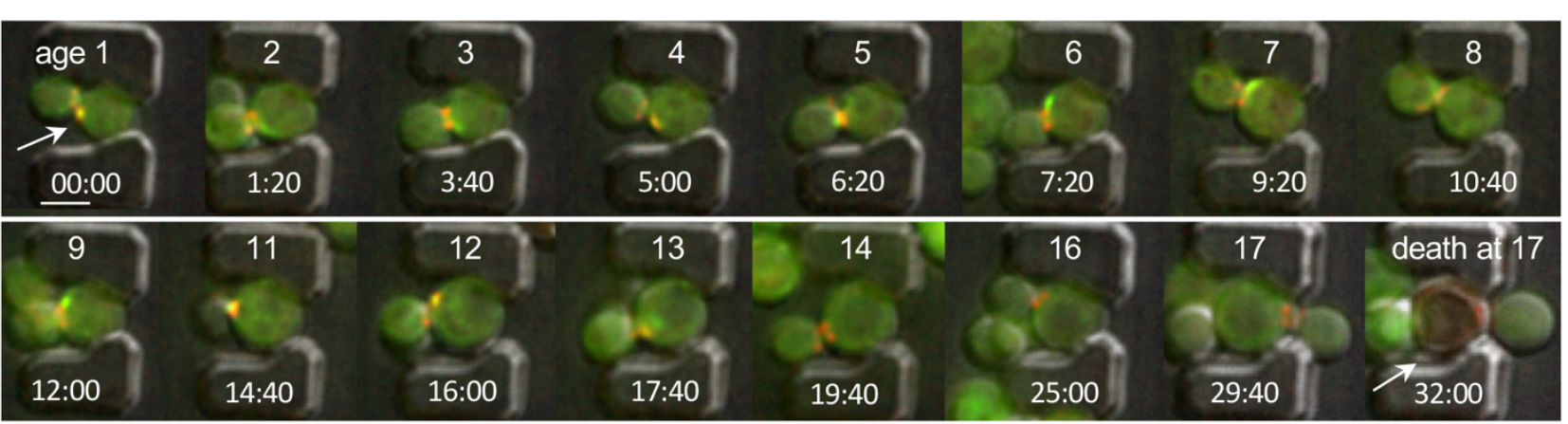

C

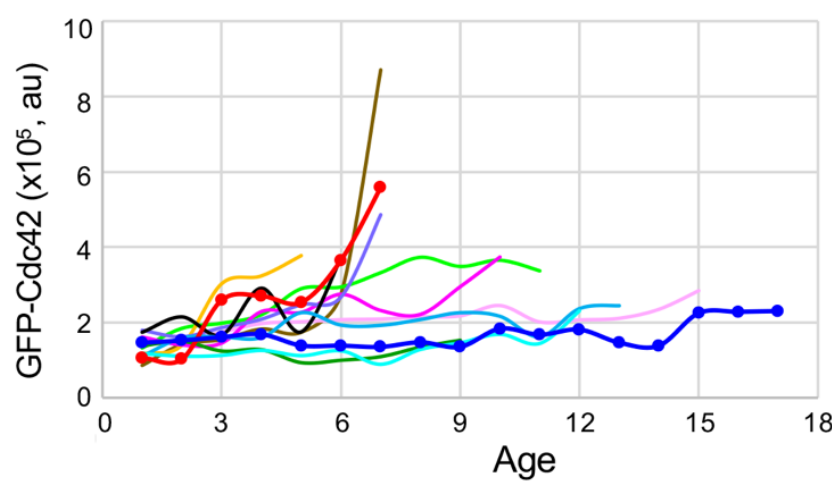

Figure 4. Overexpression of $\mathrm{Cdc42}$ causes premature aging

A (a). The level of GFP-Cdc42 in young cells (age 1) of the GFP-CDC42 and GFP-CDC42ov strains. Dots represent mean global intensity of GFP-Cdc42 in individual cells $(\mathrm{n}=21, G F P-C D C 42 ; \mathrm{n}=39$, $C D C 42 o v)$. Horizontal lines and error bars denote mean \pm SEM, and $p$ values are shown from unpaired two-tailed t-test. 
A (b). RLS estimation by microfluidic imaging at $29^{\circ} \mathrm{C}$. WT $(n=70)$ carries the endogenous $C D C 42$, and the CDC42ov strain $(\mathrm{n}=90)$ carries GFP-CDC42 X 8 on the chromosome. Graph shows the percentage of surviving cells after the indicated number of cell divisions (mean $\pm \mathrm{SEM}$ ). The median number of cell divisions that each strain has undergone before senescence or death is indicated in parentheses. $p<0.0001$ (WT vs CDC42ov); Log-rank (Mantel-Cox) test. Note: These strains are in the same background that is different from those used in Figure 1 (see Table S1).

B. Microfluidic images of GFP-CDC42ov (also expresses Cdc3-mCherry) are shown at selected time points. Numbers at the top denote ages, and the numbers at the bottom indicate the relative time (hr: min) from the first image at age 1. Fluorescent images were deconvolved. Scale bars: $5 \mu \mathrm{m}$. (a) A representative cell showing characteristics of premature aging and elevation of GFP-Cdc42 near senescence. White arrows mark a mother cell, which has likely entered senescence at age 6 7. Blue arrows mark a bud from the 7th daughter cell, which died at its first cell division. Daughter cells are larger than mother at the 6th and 7th divisions. (b) A representative cell with a longer RLS than the average undergoes normal asymmetric cell divisions until death at age 17. (c) Total intensity of GFP$\mathrm{Cdc} 42$ during cytokinesis $\sim \mathrm{G} 1$ is plotted at each age of GFP-CDC42ov cells. Colored lines represent individual mother cells from age 1 to death or senescence. Red and blue lines with markers represent the cells shown in (a) and (b), respectively. 


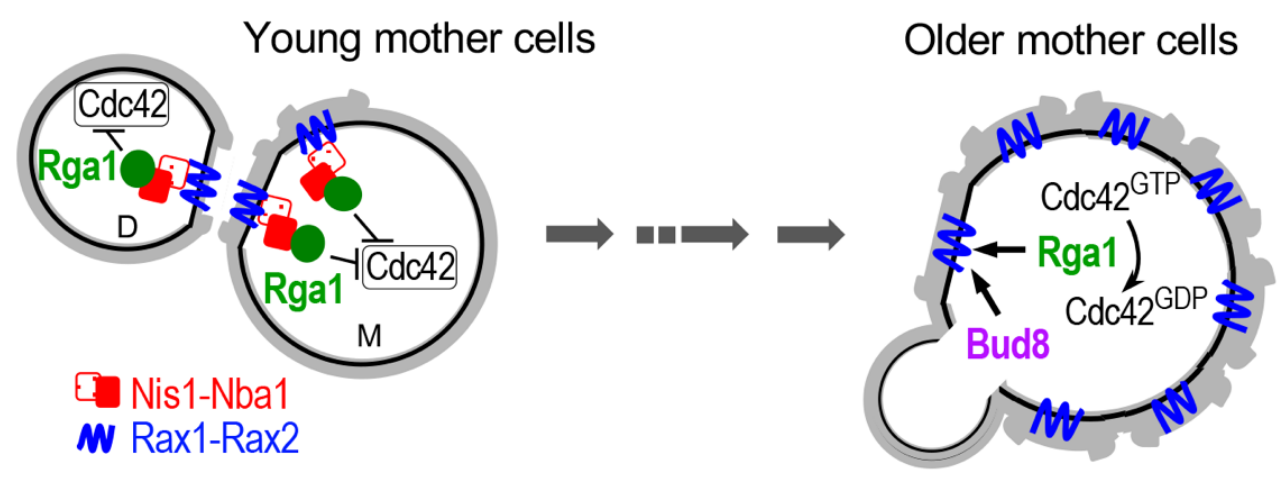

Figure 5. A model. In newborn daughter cells and young mother cells, negative polarity factors such as Rga1, which is recruited to CRMs via Nis 1 and Nba1, play a major role in preventing Cdc42 activity at the cell cortex. Bud8, which localizes to CRMs and the distal pole, may counteract negative polarity signaling in older cells, which have more CRMs, leading to an elevation of Cdc 42 activity at the overall cell cortex. The Nis1-Nba1 complex is omitted in older cells for simplicity. 
a

Haploid $m N G-B U D 8$, every $10 \mathrm{~min}$

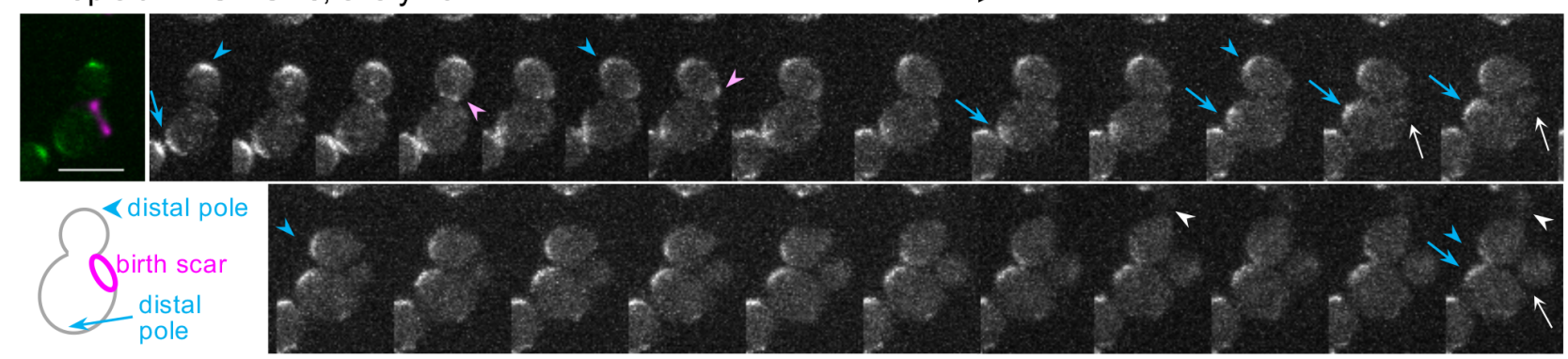

b
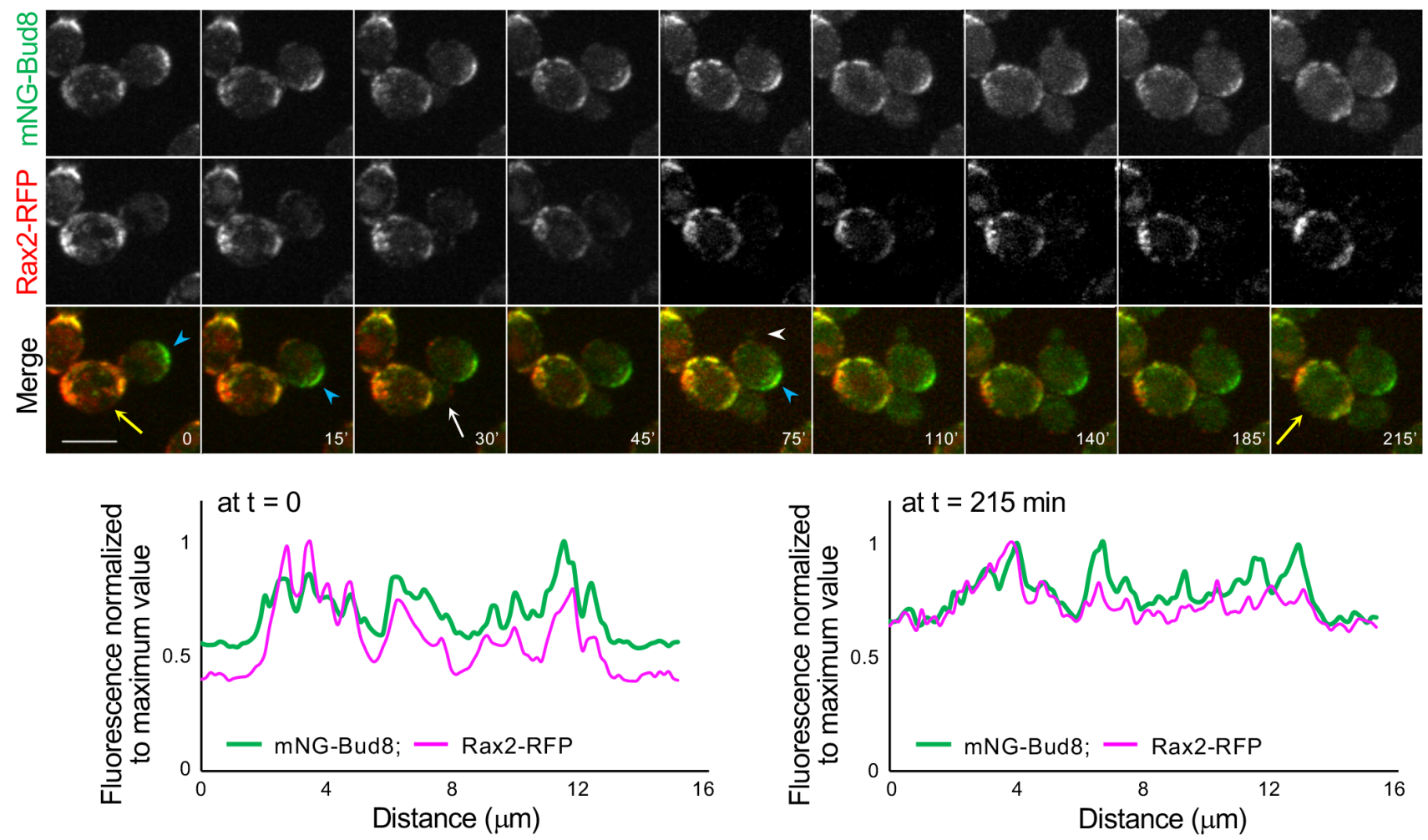

Figure S1. Time-lapse images of mNG-Bud8 and Rax2-tdTomato

a. Localization of mNG-Bud8 in a haploid WT cell, which is pre-stained with WGA-Texas Red, at $30^{\circ} \mathrm{C}$. Blue arrows and arrowheads mark the distal pole of mother and bud (which became a newborn daughter cell), respectively. Pink arrowheads mark Bud8 signal at the proximal pole of daughter cell. White arrow and arrowheads mark new buds formed from the initial mother and daughter cells, respectively. Scale bar: $5 \mu \mathrm{m}$.

b. Time-lapse images of mNG-Bud8 and Rax2-tdTomato. Blue arrowheads mark distal pole of a bud (and a newborn daughter cell). A white arrow and a white arrowhead mark new bud emergence in mother and daughter cell, respectively. Colocalization of Bud8 and Rax 2 in mother cells appears in multiple puncta at CRMs. Plots of normalized fluorescence intensity profiles of Bud8 and Rax2 along the periphery of the mother cell at selected timepoints (marked with yellow arrows) are shown below. Scale bar: $5 \mu \mathrm{m}$. 
a

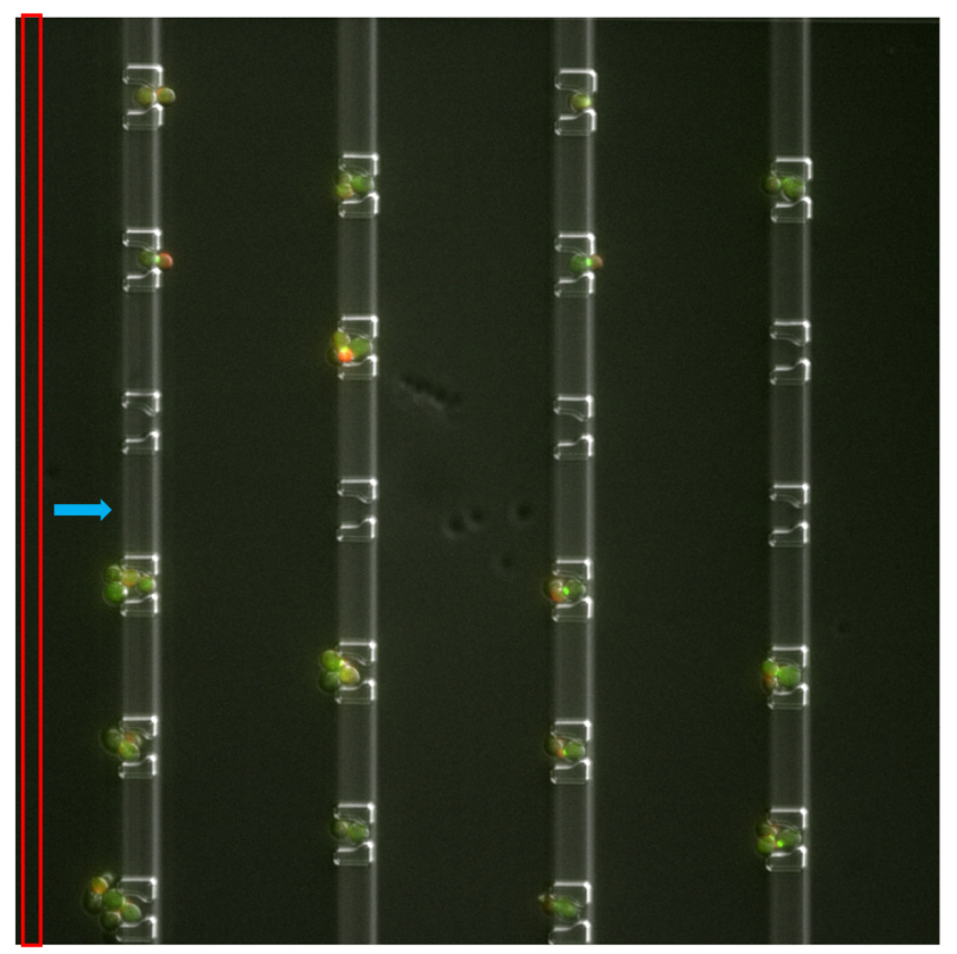

C

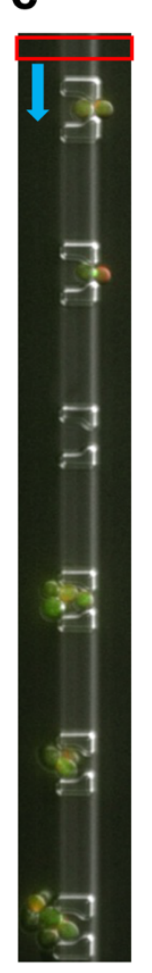

f b

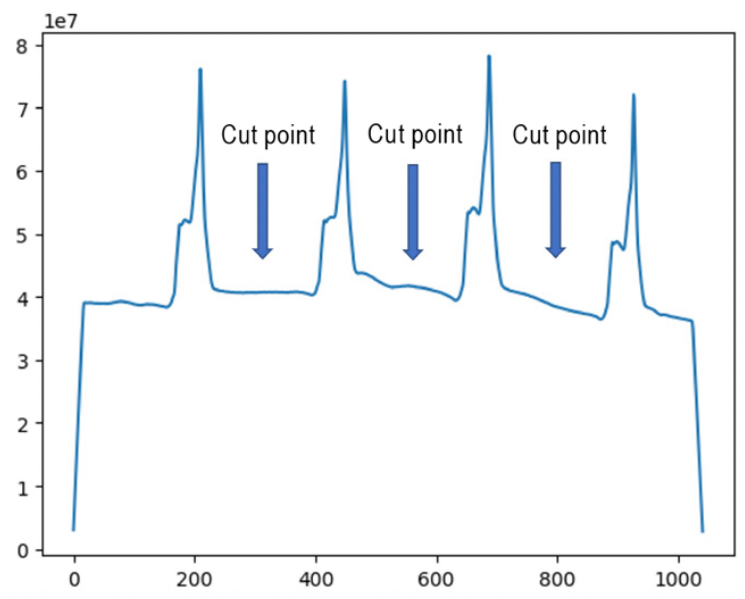

e

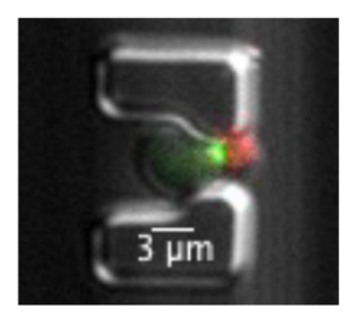

d

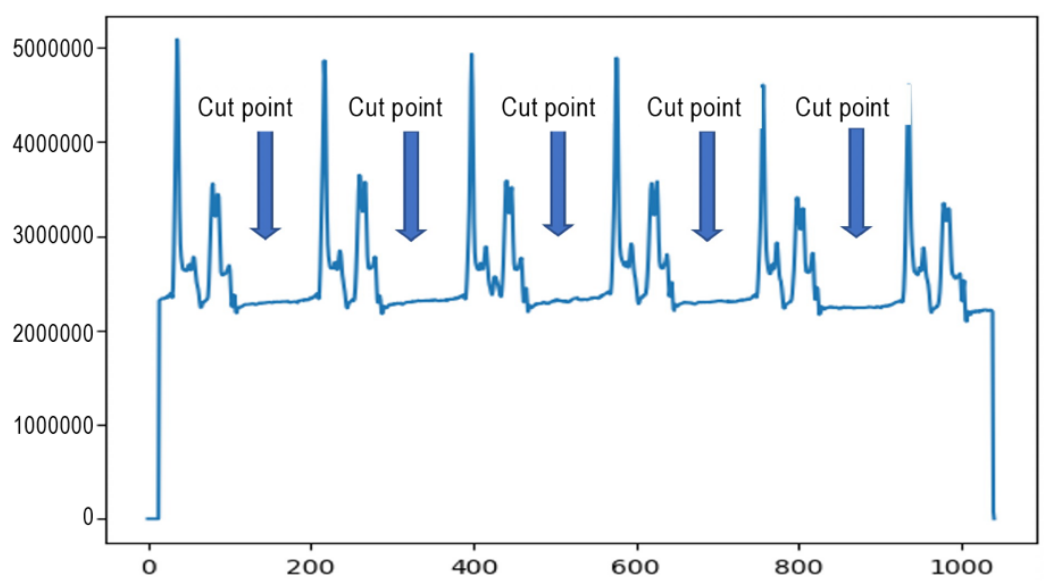

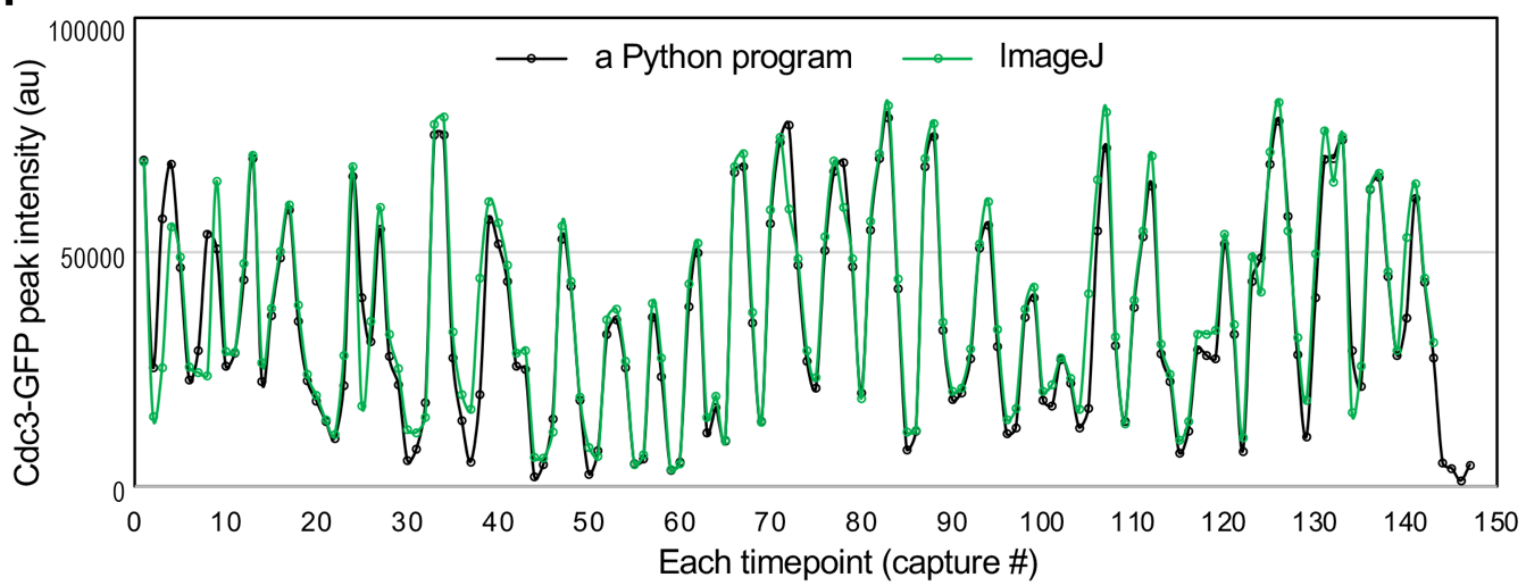

Figure S2. A Python program and RLS estimation 
To crop the whole image (a), first, a window (red box) moving horizontally (blue arrow) is used. The average pixel intensity for the window is shown in (b). Cutting between peaks in (b) generates image slices shown in (c). Next, sliding a window (red box in c) vertically generates the average intensity shown in (d). Cutting between peaks as marked in (d) results in a single cell image (e) and the intensity values of each fluorescence channel. A cell (expressing PBD-tdTomato and Cdc3-GFP) loaded in a single trap is shown in (e), and the highest pixel intensity of Cdc3-GFP in a WT cell is quantified at each time point by either the Python program or ImageJ. The number of valleys of this graph represents the number of cell divisions that this cell has undergone until death. 